\title{
Receptivity of Hypersonic Boundary Layers Due to Acoustic Disturbances over Blunt Cone
}

\author{
K. KARA* \\ Department of Aerospace Engineering \\ Old Dominion University, Norfolk, VA, 23529 \\ P. Balakumar ${ }^{\dagger}$ \\ Flow Physics and Control Branch \\ NASA Langley Research Center, Hampton, VA 23581 \\ O. A. Kandil \\ Department of Aerospace Engineering \\ Old Dominion University, Norfolk, VA, 23529
}

\begin{abstract}
The transition process induced by the interaction of acoustic disturbances in the freestream with boundary layers over a 5-degree straight cone and a wedge with blunt tips is numerically investigated at a free-stream Mach number of 6.0. To compute the shock and the interaction of shock with the instability waves the Navier-Stokes equations are solved in axisymmetric coordinates. The governing equations are solved using the $5^{\text {th }}$-order accurate weighted essentially non-oscillatory (WENO) scheme for space discretization and using third-order total-variation-diminishing (TVD) Runge-Kutta scheme for time integration. After the mean flow field is computed, acoustic disturbances are introduced at the outer boundary of the computational domain and unsteady simulations are performed. Generation and evolution of instability waves and the receptivity of boundary layer to slow and fast acoustic waves are investigated. The mean flow data are compared with the experimental results. The results show that the instability waves are generated near the leading edge and the non-parallel effects are stronger near the nose region for the flow over the cone than that over a wedge. It is also found that the boundary layer is much more receptive to slow acoustic wave (by almost a factor of 67) as compared to the fast wave.
\end{abstract}

\section{Introduction}

$\mathrm{T}$ ransition from laminar to turbulent state in shear flows occurs due to evolution and interaction of different disturbances inside the shear layer. Though there are several mechanisms and routes to go from a laminar to a turbulent state, most of them are generally follow these fundamental processes; a) receptivity b) linear instability c) nonlinear instability and saturation d) secondary instability and breakdown to turbulence.

In the receptivity process, unsteady disturbances in the environment such as acoustic waves and turbulence interact with the inhomogeneities in the geometry such as roughness and generate instability waves inside the shear layer. In quiet environments, the initial amplitudes of these instability waves are small compared to any characteristic velocity and length scales in the flow. In the linear instability stage, the amplitudes of these instability waves grow exponentially downstream and this process is governed by the linearized Navier-Stokes equations. Further downstream, the amplitudes of the disturbances become large and the nonlinear effects inhibit the exponential growth and the amplitude of the waves eventually saturate. In the next stage, these finite amplitude saturated disturbances become unstable and two- and/or three dimensional disturbances. This is called the secondary instability and beyond this stage the spectrum broadens, due to complex interactions and further instabilities, and the

\footnotetext{
${ }^{*}$ Graduate Research Assistant, Student Member AIAA

${ }^{\dagger}$ Research Scientist, Member AIAA

${ }^{\ddagger}$ Professor \& Eminent Scholar, Associate Fellow AIAA
} 
flow becomes turbulent in a short distance downstream. In this paper we are concerned with the receptivity to slow and fast acoustic waves and the linear instability of hypersonic boundary layers over a cone and a wedge.

\section{Literature Review}

There have been a number of investigations conducted on the interaction of acoustic waves with supersonic boundary layers. The interactions of acoustic waves with a supersonic boundary layer using inhomogeneous stability equations were investigated by Mack $^{1}$ and Gaponov ${ }^{2}$. One important finding was that due to the interaction, the acoustic waves excite disturbances inside boundary-layer, which are much larger than that in the free stream. The interaction of stream acoustic waves with a non-parallel boundary layer was studied by Gaponov and Smorodsky ${ }^{3}$. The analysis and the calculations showed that the disturbances inside the boundary layer reach values significantly higher compared to that in the free-stream. It was also observed that there exists a critical Reynolds number where this excitation is the highest.

For a supersonic boundary layer with sufficiently high Mach number to allow both first and second Mack modes ${ }^{4}$, Fedorov and Khoklov ${ }^{5}$ considered boundary layer response to both the fast and slow acoustic waves. The boundary layer modes excited near the leading edge by the two acoustic waves can be referred to as Mode $\mathrm{F}$ and Mode S, for convenience. The work of Fedorov and Khoklov ${ }^{6}$ and Fedorov ${ }^{7}$ identified two receptivity mechanisms in this Mach number regime: (1) leading-edge receptivity and (2) inter-modal exchange between Mode F and Mode S. For adiabatic wall, Federov ${ }^{7}$ found that receptivity to slow acoustic waves could be as much as 50 times the receptivity via the fast acoustic waves. Thus, the leading edge receptivity via the slow mode excitation is much stronger than in the case of inter-modal exchange. According to Fedorov ${ }^{7}$, this receptivity mechanism may gain significance in the highly cooled boundary layers.

Ma and Zhong ${ }^{8,9,10}$ performed direct numerical simulation for a Mach 4.5 flat-plate boundary layer to investigate receptivity to fast and slow acoustic waves and the mechanisms of inter-modal exchange. They employed fifth-order accurate shock fitting method to solve the governing equations. Egorov, Fedorov and Soudakov ${ }^{11}$ investigated a similar problem at a Mach number of 6.0 whereby simulation of receptivity to slow and fast acoustic waves and the effect of incidence angle on the receptivity were studied.

The transition process induced by the interaction of acoustic disturbances in the free stream is numerically investigated for a boundary layer over a flat plate with a blunted leading edge at a free stream Mach number of 3.5 by Balakumar ${ }^{12}$. The governing equations are solved using $5^{\text {th }}$-order accurate weighted essentially non-oscillatory (WENO) scheme for space discretization and $3^{\text {rd }}$-order TVD Runge-Kutta scheme for time integration. Balakumar ${ }^{13}$ also investigated the receptivity of boundary layers over blunt flat plates and wedges at a free stream Mach number of 3.5 and at a high Reynolds number of $10^{6}$ /inch. The linear stability result of his work showed that the bluntness has a strong stabilizing effect on the stability of two dimensional boundary layers. It was also revealed that the boundary layers on blunt wedges are far more stable than on blunt flat plates. Malik and Balakumar ${ }^{14}$ investigated the receptivity of supersonic boundary layers to acoustic disturbances at a free stream Mach number 4.5. The results showed that the instability waves are generated near the leading edge region and that the boundary layer is much more receptive to slow acoustic waves by almost a factor of 20 compared to fast acoustic waves. The effect of the acoustic wave incidence angle was also investigated and it was found that the receptivity of the boundary layer on the wind ward side decreases when the incidence angle is increased.

An experimental investigation was conducted on a 5-degree half-angle cone in a conventional Mach 6 wind tunnel by Horvath et $\mathrm{al}^{15}$ to examine the effects of facility noise on boundary layer transition. They checked the influence of tunnel noise on the transition onset points by comparing transition locations determined from their test to that previously obtained in a Mach 6 low disturbance quiet tunnel.

Here, we employ a fifth order weighted essentially non-oscillatory (WENO) scheme for spatial discretization and use third order total variation diminishing (TVD) Runge-Kutta scheme for time integration to solve for the hypersonic boundary layer receptivity problem. For this study, we have selected the flow conditions and geometry used by Horvath et $\mathrm{al}^{15}$. Cone has a small nose radius $\left(\mathrm{R}_{\mathrm{n}}=0.001\right.$-in $)$ and the flow around the leading edge is resolved by using a sufficiently dense grid. We assume adiabatic wall conditions for steady flow computations. The objectives are to understand the receptivity process near the leading edge of a cone and to estimate the receptivity coefficient of the instability waves generated near the leading edge. Computations are performed to determine whether the slow or the fast acoustic waves are more efficient in generating the instability waves. Also comparisons of computed shock stand off distances with the experimental results are shown. To compare the receptivity process 
between the axisymmetric and two-dimensional geometries computations are performed for the hypersonic flows over a cone and a wedge.

\section{Governing Equations}

The equations solved are the conservative unsteady compressible two-dimensional axisymmetric Navier-Stokes equations

$$
\frac{\partial Q}{\partial t}+\frac{\partial F}{\partial x}+\frac{\partial G}{\partial y}=\frac{\partial F_{v}}{\partial x}+\frac{\partial G_{v}}{\partial y}+S
$$

where $\mathrm{Q}$ is the solution flow field vector, $\mathrm{F}$ and $\mathrm{G}$ are the axial- and radial-direction inviscid flux vectors given by

$$
Q=\left[\begin{array}{c}
\rho \\
\rho u \\
\rho v \\
\rho E
\end{array}\right] \quad F=\left[\begin{array}{c}
\rho u \\
\rho u^{2}+p \\
\rho u v \\
(\rho E+p) u
\end{array}\right] \quad G=\left[\begin{array}{c}
\rho v \\
\rho v u \\
\rho v^{2}+p \\
(\rho E+p) v
\end{array}\right]
$$

and $F_{v}$ and $G_{v}$ are the axial- and radial-direction viscous and heat conduction flux vectors given by

$$
F_{v}=\left[\begin{array}{c}
0 \\
\tau_{x x} \\
\tau_{x y} \\
u \tau_{x x}+v \tau_{x y}-q_{x}
\end{array}\right] \quad G_{v}=\left[\begin{array}{c}
0 \\
\tau_{y x} \\
\tau_{y y} \\
u \tau_{y x}+v \tau_{y y}-q_{y}
\end{array}\right]
$$

The vector $\mathrm{S}$ contains viscous flux and source terms associated with the axisymmetric geometry. In 2D formulation, $\mathrm{S}$ will be zero and shear stresses and heat fluxes are

$$
\begin{aligned}
& S=0 \\
& \tau_{x x}=\frac{2}{3} \frac{\mu}{\operatorname{Re}}\left(2 \frac{\partial u}{\partial x}-\frac{\partial v}{\partial y}\right) \quad \tau_{x y}=\frac{\mu}{\operatorname{Re}}\left(\frac{\partial u}{\partial y}+\frac{\partial v}{\partial x}\right) \quad \tau_{y y}=\frac{2}{3} \frac{\mu}{\operatorname{Re}}\left(2 \frac{\partial v}{\partial y}-\frac{\partial u}{\partial x}\right) \\
& q_{x}=-\frac{\gamma}{(\gamma-1) \operatorname{Pr} \operatorname{Re}} \frac{\partial T}{\partial x} \quad q_{y}=-\frac{\gamma}{(\gamma-1) \operatorname{Pr} \operatorname{Re}} \frac{\partial T}{\partial y}
\end{aligned}
$$

In axisymmetric formulation source term, shear stresses and heat fluxes have the following form

$$
\begin{aligned}
& S=\frac{1}{y}\left\{\left[\begin{array}{c}
0 \\
\tau_{y x} \\
\tau_{y y}-\tau_{\theta \theta} \\
u \tau_{y x}+v \tau_{y y}-q_{y}
\end{array}\right]-\left[\begin{array}{c}
\rho v \\
\rho u v \\
\rho v^{2} \\
(\rho E+p) v
\end{array}\right]\right\} \\
& \tau_{x x}=\frac{2}{3} \frac{\mu}{\operatorname{Re}}\left(2 \frac{\partial u}{\partial x}-\frac{\partial v}{\partial y}-\frac{v}{y}\right) \quad \tau_{y y}=\frac{2}{3} \frac{\mu}{\operatorname{Re}}\left(2 \frac{\partial v}{\partial y}-\frac{\partial u}{\partial x}-\frac{v}{y}\right) \\
& \tau_{\theta \theta}=\frac{2}{3} \frac{\mu}{\operatorname{Re}}\left(2 \frac{v}{y}-\frac{\partial u}{\partial x}-\frac{\partial v}{\partial y}\right) \quad \tau_{x y}=\frac{\mu}{\operatorname{Re}}\left(\frac{\partial u}{\partial y}+\frac{\partial v}{\partial x}\right) \\
& q_{x}=-\frac{\gamma}{\gamma} \frac{q_{y}}{(\gamma-1) \operatorname{Pr} \operatorname{Re}} \frac{-\frac{\partial T}{\partial x}}{(\gamma-1) \operatorname{Pr} \operatorname{Re} \frac{\partial T}{\partial y}}
\end{aligned}
$$

American Institute of Aeronautics and Astronautics 
There exist a singularity along the axis of symmetry $y=0$. After applying L'Hopital rule and taking limit as $y$ goes to zero using the symmetry conditions $\frac{\partial u}{\partial y}=\frac{\partial \rho}{\partial y}=\frac{\partial T}{\partial y}=\frac{\partial p}{\partial y}=0, v=0$ at $\mathrm{y}=0$, source term along the axis $\mathrm{y}=0$ becomes

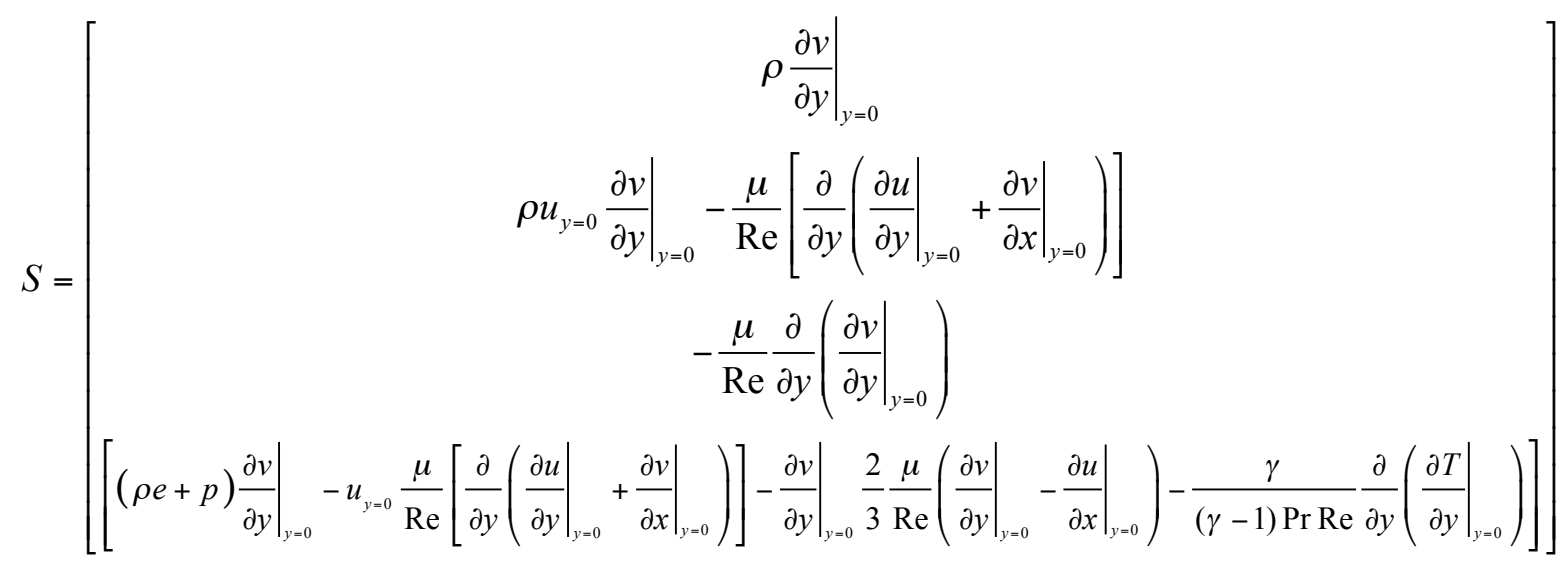

Applying same procedure to shear stresses and heat fluxes we get new expressions for the shear stresses at the axis $\mathrm{y}=0$.

$$
\begin{array}{ll}
\tau_{x x}=\frac{2}{3} \frac{\mu}{\operatorname{Re}}\left[\left.2\left(\frac{\partial u}{\partial x}-\frac{\partial v}{\partial y}\right)\right|_{y=0}\right] & \tau_{y y}=\left.\frac{2}{3} \frac{\mu}{\operatorname{Re}}\left(\frac{\partial v}{\partial y}-\frac{\partial u}{\partial x}\right)\right|_{y=0} \\
\tau_{\theta \theta}=\left.\frac{2}{3} \frac{\mu}{\operatorname{Re}}\left(\frac{\partial v}{\partial y}-\frac{\partial u}{\partial x}\right)\right|_{y=0} & q_{y}=0 \\
q_{x}=-\left.\frac{\gamma}{(\gamma-1) \operatorname{Pr} \operatorname{Re}} \frac{\partial T}{\partial x}\right|_{y=0} &
\end{array}
$$

Here $(\mathrm{x}, \mathrm{y})$ are the two-dimensional and $(\mathrm{x}, \mathrm{y}, \theta)$ are the axisymmetric coordinates and $(u, v)$ are the corresponding velocity components, $\rho$ is the density, $p$ is the pressure. $E$ is the total energy given by

$$
\begin{aligned}
& E=e+\frac{u^{2}+v^{2}}{2} \\
& e=c_{v} T, \quad p=\rho R T
\end{aligned}
$$

Here $e$ is the molecular internal energy and $T$ is the temperature.

The viscosity $(\mu)$ is computed using Sutherland's law and the coefficient of conductivity $(k)$ is given in terms of Prandtl number $(P r)$. The variables $\rho, p, T$ and velocity are non-dimensionalised by their corresponding reference variables $\rho_{\infty}, p_{\infty}, T_{\infty}$ and $\sqrt{R T}$ respectively. The reference value for lengths is computed by $\sqrt{v_{\infty} x_{0} / U_{\infty}}$, where $x_{0}$ is the location of the beginning of the computational domain in the stream wise direction. For the computation, the equations are transformed from physical coordinate system $(\mathrm{x}, \mathrm{y})$ to the computational curvilinear coordinate system $(\xi, \eta)$ in a conservative manner and the governing equations become

$$
\frac{\partial \bar{Q}}{\partial t}+\frac{\partial \bar{F}}{\partial \xi}+\frac{\partial \bar{G}}{\partial \eta}=\frac{\partial \bar{F}_{v}}{\partial \xi}+\frac{\partial \bar{G}_{v}}{\partial \eta}+\bar{S}
$$

The components of the flux in the computational domain related to the flux in the physical domain by 


$$
\bar{Q}=\frac{Q}{J}, \quad \bar{F}=\frac{J}{|J|} F, \quad \bar{G}=\frac{J}{|J|} G, \quad \bar{F}_{v}=\frac{J}{|J|} F_{v}, \quad \bar{G}_{v}=\frac{J}{|J|} G_{v}, \bar{S}=\frac{J}{|J|} S \text { and } J=\left[\frac{\partial(\xi, \eta)}{\partial(x, y)}\right]
$$

\section{Solution Algorithm}

The governing equations are solved using $5^{\text {th }}$ order accurate weighted essentially non-oscillatory (WENO) scheme for space discretization and using third order total-variation-diminishing (TVD) Runge-Kutta scheme for time integration. These methods are suitable in flows with discontinuities or high gradient regions. These schemes solve the governing equations discretely in a uniform structured computational domain in which flow properties are known at the grid nodes. WENO scheme approximate the spatial derivatives in a given direction to a higher order at the nodes, using the neighboring nodal values in that direction. TVD-RK scheme integrates the resulting equations in time to get the point values as a function of time. Since the spatial derivatives are independent of the coordinate directions, the method can easily add other dimensions. It is well known that approximating a discontinuous function by a higher order (two or more) polynomial generally introduces oscillatory behavior near the discontinuity, and this oscillation increases with the order of the approximation. The essentially non oscillatory (ENO) and the improvement of these WENO methods are developed to keep the higher order approximations in the smooth regions and to eliminate or suppress the oscillatory behavior near the discontinuities. They are achieved by systematically adopting or selecting the stencils based on the smoothness of the function, which is being approximated. Shu ${ }^{16}$ explains the WENO and the TVD methods and the formulas. Atkins ${ }^{17}$ gives the application of ENO method to the N-S equations. Balakumar ${ }^{18}$ describes in detail the solution method implemented in this computation.

At the outflow boundary, extrapolation boundary condition is used. At the wall, the simulation uses viscous conditions for the velocities and an adiabatic condition for the temperature. The density is computed from the continuity equation. In the mean flow computations, the simulation prescribes the free-stream values at the outer boundary, which lies outside the bow shock. The blunt cone is assumed to align with the free stream. In the unsteady computations, it superimposes the acoustic perturbations to the uniform mean flow at the outer boundary. The procedure is to first compute the steady mean flow by performing unsteady computations using a variable time step until the maximum residual reaches a small value $\sim 10^{-11}$. These computations use a CFL number of 0.2 . The next step is to introduce unsteady disturbances at the outer boundary of the computational domain and to perform time accurate computations to investigate the interaction and evolution of these disturbances to downstream. Since we use very fine spatial grid to resolve the leading edge region, these computations require very small time step which is taken as the minimum time step allowable for a CFL number of 0.2 .

The grid is generated using analytical formulae. The grid stretches in the $\eta$ direction close to the wall and is uniform outside of the boundary layer. In the $\xi$ direction, the grid is symmetric about the leading edge and very fine near the nose and is uniform in the flat region. The outer boundary that lies outside the shock follows a parabola so the boundary layer growth could be captured accurately. The computational domain extends from $x=-0.015$ to 9.0 inches in the axial direction in this computation. Calculations were performed using a grid $\left(32 x\left[\begin{array}{lll}127 & x & 256\end{array}\right]\right.$

1 million) depending on the size of the domain. Due to the very fine grid requirement near the nose, the allowable time step is very small and the computations become very expensive to simulate the unsteady computations in the entire domain.

The acoustic field that impinges on the outer boundary is taken to be in the following form.

$$
p^{\prime}=\operatorname{Real}\left\{\tilde{p} e^{i \alpha_{a c} x \pm i \varepsilon_{a c} y-i \omega t}\right\}
$$

Here $\alpha_{\mathrm{ac}}, \varepsilon_{\mathrm{ac}}$ are the $\mathrm{x}, \mathrm{y}$ wave numbers, respectively, of the acoustic wave and $\omega$ is the corresponding frequency of the acoustic disturbance. The incident angle $\theta$ of the acoustic wave is defined as

$$
\theta=\tan ^{-1} \frac{\varepsilon_{a c}}{\alpha_{a c}}
$$

and in this paper computations are performed for zero incidence angle. 


\section{Results}

Computations are performed for hypersonic flows over cone and wedge with blunt leading edge, $R_{n}=0.001$ in. Table 1 gives the flow parameters in the free stream and Fig. 1 shows the schematic diagram of the computational set up. First the mean flow field over a 5-degree straight cone with blunt nose tip is computed. After the mean flow is computed, acoustic disturbances are introduced at the outer boundary. The conditions at the boundary layer edge are given in Table 2 .

Table 1. Flow parameters for Horvath's ${ }^{15}$ wind tunnel model.

\begin{tabular}{|l|r|l|}
\hline Free stream & & \\
\hline \multicolumn{1}{|c|}{ Mach number } & $\mathrm{M}_{\infty}=$ & 6.0 \\
\hline Reynolds number & $\mathrm{Re}_{\infty}=$ & $7.8 \times 10^{6} / \mathrm{ft}$ \\
\hline Density & $\rho_{\infty}=$ & $7.059 \times 10^{-3} \mathrm{lbm} / \mathrm{ft}^{3}$ \\
\hline Velocity & $\mathrm{U}_{\infty}=$ & $3140.21 \mathrm{ft} / \mathrm{s}$ \\
\hline Reservoir Pressure & $\mathrm{P}_{0}=$ & $475 \mathrm{psi}$ \\
\hline Reservoir Temperature & $\mathrm{T}_{0}=$ & $475^{\circ} \mathrm{F}$ \\
\hline Wall temperature & Adiabatic condition \\
\hline Prandtl number & $\mathrm{Pr}=$ & 0.70 \\
\hline Ratio of specific heats & $\gamma=$ & 1.4 \\
\hline Length scale (x $\mathrm{x}_{0}=0.5$ inch) & $\sqrt{v_{\infty} x_{0} / U_{\infty}}=$ & $7.30882 \times 10^{-5} \mathrm{ft}$ \\
\hline
\end{tabular}

Table 2. Conditions at the edge of the boundary layer.

\begin{tabular}{|l|c|c|}
\hline (Var.) $)_{\text {edge }} /(\text { Var. })_{\infty}$ & Cone & Wedge \\
\hline Mach Number & 0.932 & 0.885 \\
\hline Pressure Ratio & 1.560 & 2.069 \\
\hline Density Ratio & 1.372 & 1.663 \\
\hline Temperature Ratio & 1.137 & 1.244 \\
\hline
\end{tabular}

\section{Validation of Axisymmetric Solver.}

To validate the axisymmetric solver a series of comparison with the data available in the literature had been done. Flow field around a hemisphere-cone has been computed and results have compared with Mair's ${ }^{19}$ experiment. Table 3 gives the flow parameters and Fig. 2 shows the comparison of the flow field.

Table 3. Flow parameters for Mair's ${ }^{19}$ wind tunnel model.

\begin{tabular}{|l|r|l|}
\hline Free stream & & \\
\hline \multicolumn{1}{|c|}{ Mach number } & $\mathrm{M}_{\infty}=$ & 1.96 \\
\hline Reynolds number & $\mathrm{Re}_{\infty}=$ & $1.3 \times 10^{7} / \mathrm{m}$ \\
\hline Density & $\rho_{\infty}=$ & $0.2922 \mathrm{~kg} / \mathrm{m}^{3}$ \\
\hline Velocity & $\mathrm{U}_{\infty}=$ & $511.76 \mathrm{~m} / \mathrm{s}$ \\
\hline Reservoir Pressure & $\mathrm{P}_{0}=$ & $14230.9 \mathrm{~N} / \mathrm{m}^{2}$ \\
\hline Reservoir Temperature & $\mathrm{T}_{0}=$ & $169.65^{\circ} \mathrm{K}$ \\
\hline Wall temperature & Adiabatic condition \\
\hline Prandtl number & $\mathrm{Pr}=$ & 0.70 \\
\hline Ratio of specific heats & $\gamma=$ & 1.4 \\
\hline Length scale $\left(\mathrm{x}_{0}=0.5 \mathrm{~m}\right)$ & $\sqrt{v_{\infty} x_{0} / U_{\infty}}=$ & $6.5 \times 10^{-3} \mathrm{~m}$ \\
\hline
\end{tabular}

Ambrosio and Wortman ${ }^{20}$ used experimental results to develop correlations for shock stand off distances as a function of Mach number. Figure 3 shows the comparison of shock stand off distances between the simulation and the predicted values from the correlation and Fig. 4 shows the comparison of shock shape at Mach 6.0 with Billing's ${ }^{21}$ correlation for cylinder-wedge and sphere-cone. It is seen the numerical simulation agrees with the correlations very well.

Horvath et. al. ${ }^{15}$ investigated the effect of bluntness on the transition onset at $\mathrm{M}=6.0$ at different Reynolds numbers for a 5 degrees cone. They verified that the bluntness delays boundary layer transition in the hypersonic 
flows. Computations were performed at three different nose radiuses as in the experiment and the wall to total temperature ratios are compared with the experimental results in Fig. 5. The figure shows a perfect match between the experimental and the simulation results. In the experiment, nose radius for the sharp cone is 0.0001 -inches and to perform a simulation with this small bluntness need very fine grid in the leading edge region and very small CFL number. To save the computational effort and time, a nose radius of $\mathrm{Rn}=0.001$-inches is chosen in this simulation. This explains the small shift in the temperature ratio between the computation and the experiment.

\section{Linear Stability}

Linear stability results for the similarity boundary layer over a cone and a wedge for the inviscid conditions at the surfaces are presented in Fig. 6. Figures 6(a) and (b) show the neutral stability diagram in (Re, F) plane for twodimensional disturbances. The figure shows the first and second mode unstable regions and the variation of the wave number with the Reynolds number. Figures 6(c) and (d) show the N-Factor curves for different frequencies. Here the variables are non-dimensionalized by the variables at the edge of boundary layer. To obtain the variables nondimensionalized by the free stream values as given in Table 1, the variables in this section should be multiplied by the appropriate factors from Table 2 . The frequency variable $\mathrm{F}$ has to be multiplied by 1.174 to obtain the values in terms of free stream values.

The neutral stability curve clearly shows the unstable first and the second mode regions for the boundary layers over the cone and wedge at a free stream Mach number 6.0. The first mode and the second mode neutral stability curves merge at a Reynolds number of $\mathrm{Re}=1600$ for the cone and they do not merge for the wedge case for these parameters. The most amplified frequencies are higher for the cone boundary layers than that for the wedge. The transition Reynolds numbers based on an N-Factor of 9 are about 3500 for the cone and about 5200 for the wedge. The most amplified frequencies are $0.85 \times 10^{-4}$ and $0.34 \times 10^{-4}$ for the cone and the wedge respectively.

\section{Acoustic Waves}

The linearized Euler equations in a uniform mean flow are:

$$
\begin{aligned}
& \frac{\partial \rho}{\partial t}+U_{0} \frac{\partial \rho}{\partial x}+\rho_{0} \frac{\partial u}{\partial x}+\rho_{0} \frac{\partial v}{\partial y}=0 \\
& \rho_{0} \frac{\partial u}{\partial t}+\rho_{0} U_{0} \frac{\partial u}{\partial x}=-\frac{\partial p}{\partial x} \\
& \rho_{0} \frac{\partial v}{\partial t}+\rho_{0} U_{0} \frac{\partial v}{\partial x}=-\frac{\partial p}{\partial y} \\
& \rho_{0} \frac{\partial w}{\partial t}+\rho_{0} U_{0} \frac{\partial w}{\partial x}=-\frac{\partial p}{\partial z} \\
& \rho_{0} c_{p} \frac{\partial T}{\partial t}+\rho_{0} U_{0} c_{p} \frac{\partial T}{\partial x}=\frac{\partial p}{\partial t}+U_{0} \frac{\partial p}{\partial x} \\
& P_{0}=\rho_{0} R T_{0} \\
& p=\rho_{0} R T+\rho R T_{0}
\end{aligned}
$$

The solution of this system can be written as

$$
\left\{\begin{array}{c}
\rho \\
u \\
v \\
w \\
T
\end{array}\right\}=\left\{\begin{array}{c}
1 / a_{0}^{2} \\
-\alpha_{a c} /\left(\rho_{0}\left(\alpha_{a c} U_{0}-\omega\right)\right) \\
-\varepsilon_{a c} /\left(\rho_{0}\left(\alpha_{a c} U_{0}-\omega\right)\right) \\
-\beta_{a c} /\left(\rho_{0}\left(\alpha_{a c} U_{0}-\omega\right)\right) \\
(\gamma-1) T_{0} / \rho_{0} a_{0}^{2}
\end{array}\right\} p
$$

Here the pressure $\mathrm{p}$ is in the form 


$$
p=p_{a m p} e^{i\left(\alpha_{a c} x+\varepsilon_{a c} y+\beta_{a c} z-\omega t\right)}
$$

The dispersion relation among the wave numbers $\alpha_{a c}, \varepsilon_{a c}, \beta_{a c}$ and the frequency $\omega$ is given by

$$
\left(\alpha_{a c} U_{0}-\omega\right)^{2}=\left(\alpha_{a c}^{2}+\varepsilon_{a c}^{2}+\beta_{a c}^{2}\right) a_{0}^{2}
$$

For acoustic disturbances with zero sweep (i.e. $\beta_{a c}=0$ ), and zero incident angle (i.e. $\theta=\tan ^{-1} \frac{\varepsilon_{a c}}{\alpha_{a c}}=0$ ) the X-wave number $\alpha_{a c}$ can be expressed as

$$
\alpha_{a c}=\frac{\omega}{\left(U_{0} \pm a_{0}\right)}
$$

The plus sign corresponds to the fast moving wave and the minus sign corresponds to the slow moving wave. The corresponding phase speeds are $c=U_{0} \pm a_{0}$. The wave number of the fast moving wave is $\alpha_{a c}<\frac{\omega}{U_{0}+a_{0}}$ and for the slow moving wave $\alpha_{a c}>\frac{\omega}{U_{0}-a_{0}}$. For $M=6.0$ and $F=1.2 \times 10^{-4}$, wave numbers of the fast and slow moving waves are $\alpha_{a c}<0.0586$ and $\alpha_{a c}>0.0821$. Table 4 gives the wave number and the wave length for the slow, the fast frequencies.

Table 4. Wave number and wave length for $F=1.2 \times 10^{-4}$.

\begin{tabular}{|c|c|c|c|}
\hline Freq $\times 10^{-4}$ & & $\alpha_{a c}$ & $\lambda-$ in \\
\hline 1.20 & Slow & 0.0821 & 0.0671 \\
\cline { 2 - 4 }$[467.79 \mathrm{kHz}]$ & Fast & 0.0586 & 0.0940 \\
\hline
\end{tabular}

\section{Mean Flow for Cone and Wedge}

Figure 7 illustrates the mean flow data for the cone and the wedge computed using the WENO code. The figures on the left show the contours for the cone and the figures on the right show the results for the wedge. Figures 7 (a) and (b) show the mean flow density contours for the entire domain extracted from the Navier-Stokes computations. As expected the bow shock for the cone is narrower than that for the wedge because of the relieving effect of axisymmetry. Figures 7 (c), (d) show the density contours and (e), (f) show the Mach contours near the leading edge for the cone and the wedge respectively. The bow shock for the cone is located at $\delta_{\text {cone }}=2.4 \times 10^{-4}$ inch upstream of the leading edge and for the wedge it is at $\delta_{\text {wedge }}=5.6 \times 10^{-4}$ inch. Beyond the expansion fan the shock angles approach inviscid shock angle of 10.6 degrees for the cone and 13.1 degrees for the wedge. Figures 7(g) and (h) show the streamline patterns. The density profiles at $\mathrm{x}=0.01,0.03,0.05,8.94$ inches. $\left(\sqrt{\operatorname{Re}_{x}}=87,151,196,2615\right)$ are plotted in Figs 8(a) and (b) in similarity coordinate for the cone and the wedge. The compressible Blasius similarity profile is also included for comparison. The boundary layer profiles approach to the similarity solutions close to $\mathrm{x}=0.1$-inch for the cone. However, the density profile for the wedge at the end of the computational domain $\mathrm{x}=9$-in still did not approach the similarity results. This implies that the bluntness effects are stronger and persist for a longer distance for flow over wedges compared to flow over cones. Figures $8(\mathrm{c})$ and (d) depicts the same mean density profiles in y coordinate. Figure 9 (a) shows the mean pressure distribution along the surface for the cone and the wedge and Fig 9(b) shows the variation of the edge Mach number. At $\mathrm{x}=8.9$-in $\mathrm{M}_{\mathrm{e}}=5.57$ and $\mathrm{T}_{\mathrm{e}}=129.61{ }^{\circ} \mathrm{R}$ for cone and $\mathrm{M}_{\mathrm{e}}=5.29$ and $\mathrm{T}_{\mathrm{e}}=141.79^{\circ} \mathrm{R}$ for wedge. It can be seen that very close to the leading edge, there exists a strong shock and that the associated compression is followed by an expansion over the leading edge and the shock approaches the inviscid solution for the cone and the wedge. 


\section{Interaction of Slow and Fast Acoustic Waves with the Cone and Wedge Boundary Layer}

After the mean flow is computed two dimensional slow and fast acoustic disturbances are separately introduced at the outer boundary computational boundary and the time accurate simulations are performed. Unsteady simulations are performed for the frequencies $\mathrm{F}=1.2 \times 10^{-4}$ and $1.4 \times 10^{-4}$ for the cone case and for the frequency $\mathrm{F}=0.85 \times 10^{-4}$ for the wedge. These frequencies give maximum amplifications within the computational domain of $\mathrm{x}=9$ inches. For the free stream parameters considered in these computations Table 1, these frequencies correspond to 331,467 and $546 \mathrm{kHz}$ respectively. To remain in the linear regime, the amplitude of the forcing freestream acoustic waves is given a small value of $\mathrm{p}_{\mathrm{ac}} / \mathrm{p}_{\infty}=2 \times 10^{-5}$. Even with this small amplitude, nonlinearity starts to develop near the computational domain for the frequency $\mathrm{F}=1.2 \times 10^{-4}$.

Figure 10 shows the results for the evolution of unsteady fluctuations obtained from the simulations for the slow wave at a fixed time for the cone case with $\mathrm{F}=1.2 \times 10^{-4}$. Figure 10(a) shows the contours of the density fluctuations in the entire domain and Fig. 10(b) depicts the results inside the boundary layer. In Figure 10(b) the surface of the cone is plotted along the $\mathrm{x}$-axis to show the expanded view of the boundary layer clearly. The perturbation field can be divided into four regions. One region is the area outside the shock where the acoustic waves propagate uniformly. The second region is the shock layer across which the acoustic waves are transmitted. The third region is the area between the shock and the boundary layer. This region consists of transmitted external acoustic field and the disturbances that are radiated from the boundary layer. The fourth region is the boundary layer where the boundary layer disturbances evolve. The figures show that the disturbances inside the boundary layer are generated near the nose region. Figure 10(b) also shows the evolution of the first mode up to $\mathrm{x} \sim 4.5$ inches and the gradual transformation of the first mode to the second mode in downstream. Another interesting observation is that the region between the boundary layer and the shock layer is quieter compared to the acoustic waves outside the shock layer. This implies that the acoustic waves are weakly transmitted through the shock. This was also observed in the flat plate simulation (of Malik and Balakumar ${ }^{15}$ ) where as the acoustic wave incidence angle is increased disturbances become quieter in the windward side.

Figure 11 shows the evolution of the wall pressure fluctuations for the cone and the wedge case. Figures 11(a) and (b) show the pressure fluctuations induced by the slow and the fast acoustic waves for the frequency $\mathrm{F}=1.2 \times 10^{-4}$ for the cone case and Fig 11(c) shows the results induced by the slow acoustic wave for the frequency $\mathrm{F}=1.4 \times 10^{-4}$. Figure 11 (d) shows the pressure fluctuation induced by the slow wave for the frequency $\mathrm{F}=0.85 \times 10^{-4}$ for the wedge case. It is to be noted that different scales are used in Fig 11 due to the different amplification in the slow and the fast mode cases. For the frequency $\mathrm{F}=1.2 \times 10^{-4}$, the maximum amplitude in the slow mode case is about 0.16 and is about 0.0025 in the fast mode case. This implies that the slow mode is more efficient; by about 67 times, in generating the instability wave inside the boundary layer compared to the fast wave. This agrees qualitatively with other simulations and analysis. One other observation is the amplification of the first mode near the leading edge region. The parallel linear computations revealed that the first mode is stable up to $\mathrm{x} \sim 3$ inch for this frequency. However, the simulation shows that the first mode disturbances are growing starting from the leading edge. Hence, the non-parallel effects are stronger in the cone case compared to the flat plate case and this yields a higher amplification ratio (about 67) between the induced flow field by the slow and the fast modes in the cone case compared to the flat plate case (about 20). Figure 11(d) shows that the amplification of the disturbances are small for the wedge case for this frequency $\mathrm{F}=0.85 \times 10^{-4}$. The maximum amplitude attained is about $2 \times 10^{-4}$ compared to 0.16 in the cone case. The first mode region in the wedge case is stable contrast to the cone case.

Figure 12 shows the amplitude of the pressure fluctuations along the wall in a log scale. This figure also includes the results from the parabolized stability equations (PSE) computations obtained for the same mean boundary layer profiles. The growth of the disturbances agrees very well with the PSE results. The figures clearly show the initial generation and the eventual exponential growth of the instability waves inside the boundary layer. The slow wave whose wavelength is closer to the wavelength of the instability wave transform into instability wave smoothly. The fast mode as was in the flat plate case initially generated the instability mode corresponding to the fast acoustic wave and then switches to the unstable second mode close to $\mathrm{x}=4.5$ inches. As was discussed earlier, the figure 12(a) shows that the first mode is growing due to the non-parallel effect. Due to the growth of the first mode starting from the nose region, it is difficult to define a receptivity coefficient in the cone case. We selected the amplitude near the nose region $\mathrm{x} \sim 1.0$ to compute the receptivity coefficient defined by the ratio between the initial amplitude of the pressure fluctuations at the wall near $\mathrm{x} \sim 1.0$ and the free stream acoustic pressure can be evaluated. For the wedge case, the first mode is decaying and a well defined neutral point is discerned. Hence, the receptivity coefficient is defined based on this amplitude. Table 5 gives the respective receptivity coefficients for the different cases. The 
receptivity coefficients for the cone cases are about 4.569 and 0.068 for the slow and the fast acoustic modes and it is about 0.77 for the slow acoustic mode for the wedge case.

$$
C_{\text {recpt }, p_{\text {wall }}}=\frac{\left(p_{\text {wall }}\right)_{n}}{p_{a c}}
$$

Table 5. Variation of receptivity coefficient for different $\mathrm{X}$ locations.

\begin{tabular}{|c|c|c|c|c|c|}
\hline & \multicolumn{2}{|c|}{ Cone } & \multicolumn{2}{c|}{ Cone } & Wedge \\
\hline$F\left(\mathrm{x} 10^{-4}\right)$ & \multicolumn{2}{|c|}{1.2} & \multicolumn{2}{|c|}{1.4} & 0.85 \\
\hline$x($ in $)$ & 1.0 & 1.5 & 1.0 & 1.5 & $\begin{array}{c}\text { Neutral Point. } \\
(\mathrm{x}=3.6)\end{array}$ \\
\hline$C_{\text {recpt }, p_{\text {wall }}, \text { Slow }}$ & 3.826 & 4.569 & 3.91 & 4.89 & 0.77 \\
\hline$C_{\text {recpt }, p_{\text {wall }}, \text { Fast }}$ & 0.057 & 0.068 & & & \\
\hline$\frac{C_{\text {recpt }, p_{\text {wall }}, \text { Slow }}}{C_{\text {recpt }, \text { pall }_{\text {wast }}, \text { Fast }}}$ & 67.12 & 67.19 & & & \\
\hline
\end{tabular}

Figure 13 shows the contours of the density fluctuations inside the boundary layer at three different streamwise locations to illustrate the structure and the evolution of the instability waves inside the boundary layer. The contours show that the disturbances are concentrated near the edge of the boundary layer and in downstream the disturbances exhibit the classical 'rope' like structures associated with the second mode.

\section{Discussion and Conclusion}

The receptivity and the stability of hypersonic boundary layers due to the interaction of two-dimensional slow and fast acoustic waves with a blunt 5-degree cone and a blunt wedge are numerically investigated at a free stream Mach number of 6.0 and at a Reynolds number of $7.8 \times 10^{6} / \mathrm{ft}$. Both steady and unsteady solutions are obtained by solving compressible Navier-Stokes equations in cylindrical coordinates using the $5^{\text {th }}$ order accurate weighted essentially non-oscillatory (WENO) scheme for space discretization and using a third-order total-variationdiminishing (TVD) Runge-Kutta scheme for time integration.

The shock stand off distances is computed for different bluntness at different Reynolds numbers and Mach numbers and is compared with experimental correlation results. The comparison showed excellent agreement between the simulation and the experimental results. The mean flow data for the cone and the wedge showed that the bluntness effect is stronger and persists longer for the wedge flows compared to the cone flows.

The unsteady simulations showed that the instability waves are generated very close to the leading edge region. The simulations for the cone showed that the first mode starts to grow starting from the leading edge due to the nonparallel effects before they grow strongly due to the unstable second mode. In the wedge case, the first mode disturbances decay first before they start to grow due to the second mode. The receptivity coefficient of the instability waves generated by the slow acoustic wave is about 4 times the amplitude of the free stream acoustic wave. It is also found that the amplitude of the instability waves generated by the slow acoustic waves is about 67 times larger than that for the case of fast acoustic waves. Therefore, forcing by slow acoustic wave is much more relevant in the transition process involved in hypersonic boundary layers. The receptivity coefficient in the wedge case is about 0.8 which is about 5 times smaller than that in the cone case. This is due to the initial growth of the first mode in the cone case compared to the decay in the wedge case and also due to the strong stabilization effect of the bluntness in the wedge case. The receptivity coefficient for a flat plate boundary layer with a bluntness of 0.0001 inches ${ }^{14}$ at a free stream Mach number of 4.5 is about 9 and the slow mode is about 20 times more efficient than the fast mode in generating the instability waves. This shows that the slow mode is much more efficient in flows over axisymmetric bodies than in two-dimensional flows.

\section{Acknowledgement}

The first and the third authors are supported by Flow Physics and Control Branch of NASA Langley Research Center for this work. 


\section{References}

${ }^{1}$ Mack, L. M., 1975, On The Application of Linear Stability Theory and The Problem of Supersonic Boundary Layer Transition, AIAA Journal, 13, 423-448.

${ }^{2}$ Gopanov, S. A., 1977, Interaction between a Supersonic Boundary Layer and Acoustic Disturbances, Journal of Fluid Dynamics, 6, 1977.

${ }^{3}$ Gopanov, S. A. and Smorodsky, B. V., 1999, Supersonic Boundary Layer Receptivity to Stream wise Acoustic Field, IUTAM Symposium, Springer-Verlag.

${ }^{4}$ Mack, L. M., 1969, Boundary Layer Stability Theory, Report 900-277 Rev. A, Jet Propulsion Lab, Pasadena, CA.

${ }^{5}$ Fedorov, A. V., and Khokhlov, A. P., 1991, Excitation of Unstable Modes in a Supersonic Boundary Layer, Fluid Dynamics, 9, 456-467.

${ }^{6}$ Fedorov, A. V., and Khokhlov, A. P., 2001, Prehistory of Instability in a Hypersonic Boundary Layer, Theoretical Computational Fluid Dynamics, 14, 359-375.

${ }^{7}$ Fedorov, A. V., Receptivity of a High Speed Boundary Layer to Acoustic Disturbances, Journal of Fluid Mechanics, 491, 101-129.

${ }^{8}$ Ma, Y. and Zhong, X., 2003, Receptivity of a Supersonic Boundary Layer over a Flat Plate. Part 1. Wave Structures and Interactions, Journal of Fluid Mechanics, 488, 31-78

${ }^{9}$ Ma, Y. and Zhong, X., 2003, Receptivity of a Supersonic Boundary Layer over a Flat Plate. Part 2. Receptivity to FreeStream Sound, Journal of Fluid Mechanics, 488, 79-121

${ }^{10}$ Ma, Y. and Zhong, X., 2005, Receptivity of a Supersonic Boundary Layer over a Flat Plate. Part 3. Effects of Different Types of Free-Stream Disturbances, Journal of Fluid Mechanics, 532, 63-109

${ }^{11}$ Egarov, I. V., Fedorov, A. V., and Soudakov, V. G., 2005, Direct Numerical Simulation of Supersonic Boundary Layer Receptivity to Acoustic Disturbances, AIAA Paper, 97.

${ }^{12}$ Balakumar, P., 2006, Stability of Supersonic Boundary Layers over Blunt Wedges, AIAA Paper, 3053.

${ }^{13}$ Balakumar, P., 2005, Transition in A Supersonic Boundary Layer Due To Acoustic Disturbances, AIAA Paper, 96.

${ }^{14}$ Malik, M. R., and Balakumar, P., 2005, Receptivity of Supersonic Boundary Layers to Acoustic Disturbances, AIAA Paper, 5027.

${ }^{15}$ Horvath, T. J., Berry, S. C., Hollis, B. R., Chang, C. C., Singer, B. A., 2002, Boundary Layer Transition On Slender Cones In Conventional And Low Disturbances Mach 6 Wind Tunnels, AIAA Paper, 2743

${ }^{16}$ Shu, C. W., 1997, Essentially Non-Oscillatory And Weighted Essentially Non-Oscillatory Schemes For Hyperbolic Conservation Laws, NASA/CR-97-206253 and ICASE Report No. 97-6.

${ }^{17}$ Atkins, H. L., 1991, High-Order ENO Methods for the Unsteady Compressible Navier-Stokes Equations, AIAA Paper, 1557.

${ }^{18}$ Balakumar, P., 2002, Stability of Hypersonic Boundary Layers Over a Compression Corner, AIAA Paper, 2848.

${ }^{19}$ Mair, W. A, 1952, Experiments on Separation of Boundary Layers on Probes in front of Blunt Nosed Bodies in a Supersonic Air Stream, Phil. Mag. Ser. 7, Vol 43, No 343, 695.

${ }^{20}$ Ambrosio, A. and Wortman, A., 1962, Stagnation Point Shock Detachment Distance for Flow around Spheres and Cylinders, ARS Journal, 32, pp. 281.

${ }^{21}$ Billig, F. S., 1967, Shock-Wave Shapes around Spherical and Cylindrical-Nosed Bodies, Journal of Spacecraft, 4, 6, 822823. 


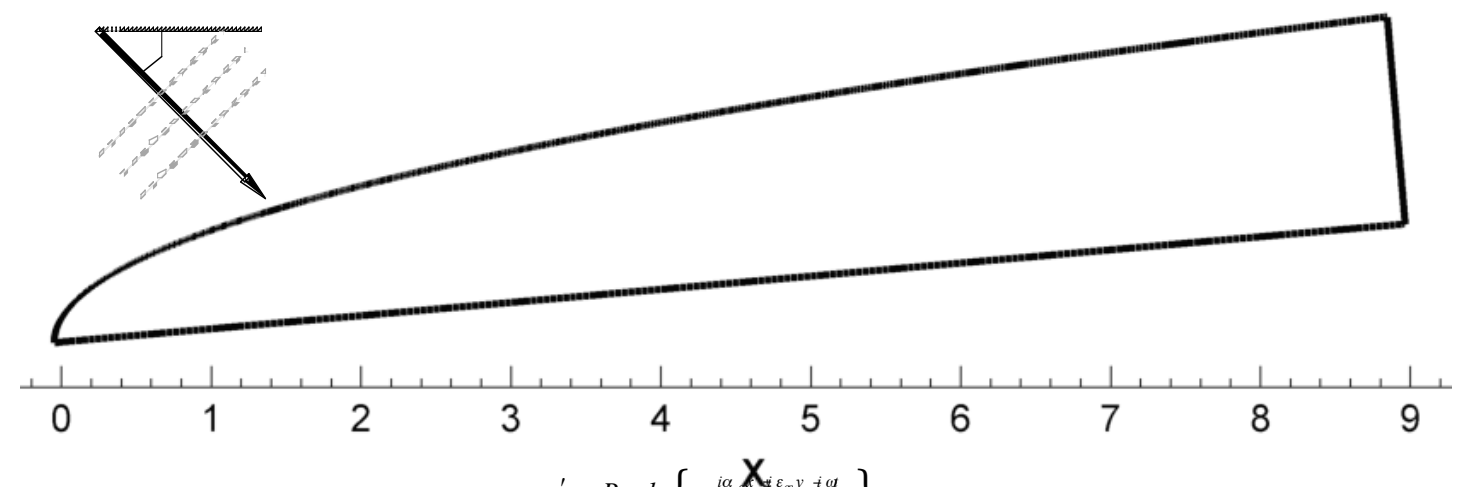

Figure 1. Schematic diagram of the computational model. Parallel computation done with 32 block (32x[127x 256] 1 million points)

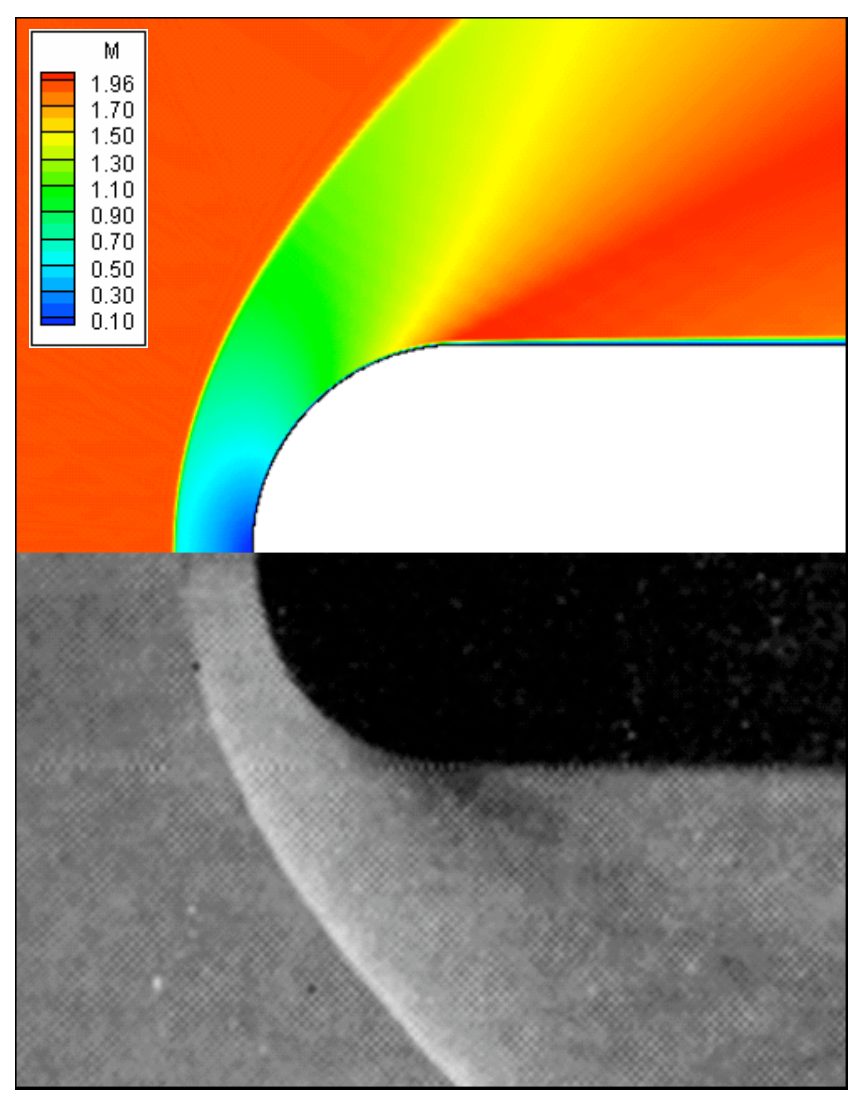

(a)
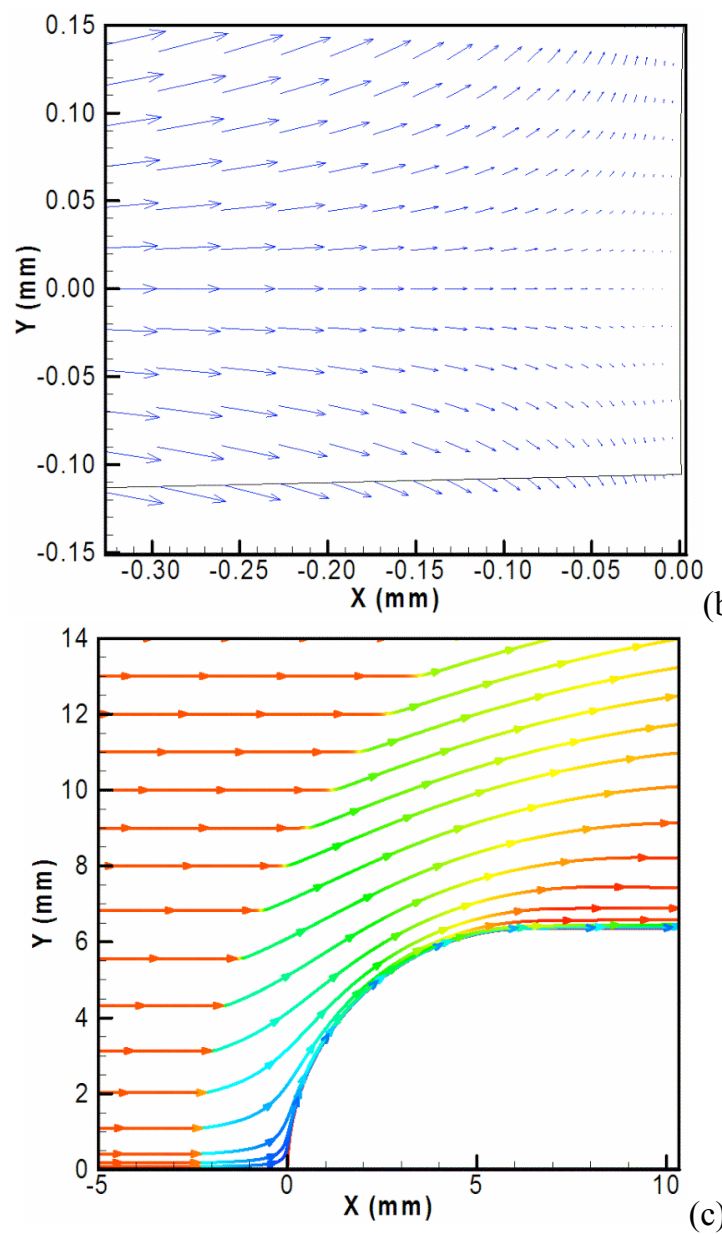

(c)

Figure 2. (a) Comparison of Mach contours with Mair's experiment ${ }^{19}$ (b) Velocity vectors near stagnation point. (c) Streamline pattern colored by Mach contours near leading edge. 


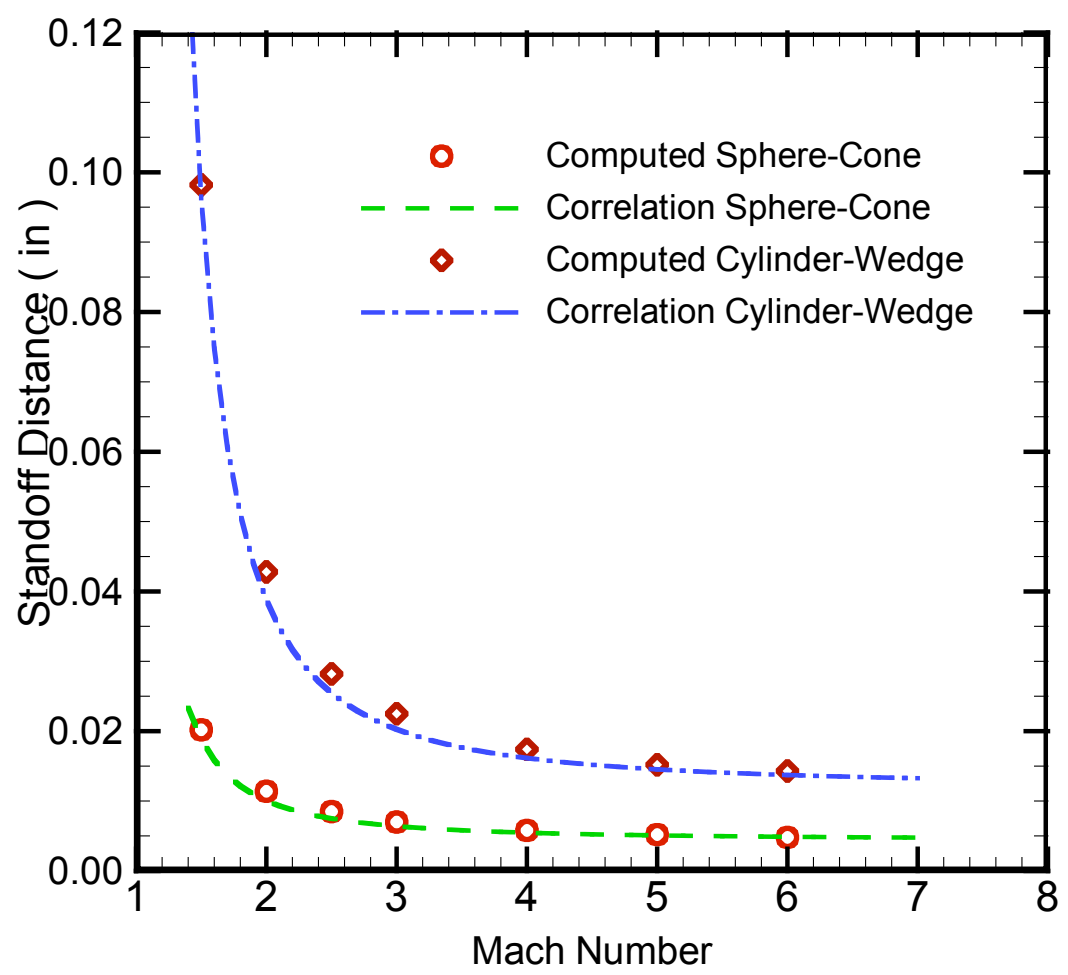

Figure 3. Comparison of computed and correlated ${ }^{20}$ bow shock standing distance for sphere-cone and cylinder-wedge.

\section{Cone}

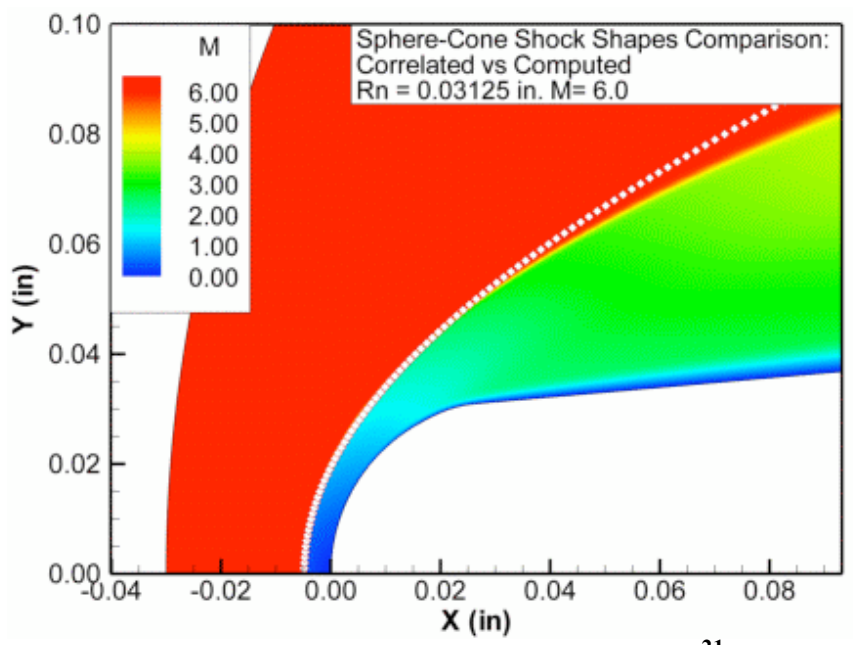

Wedge

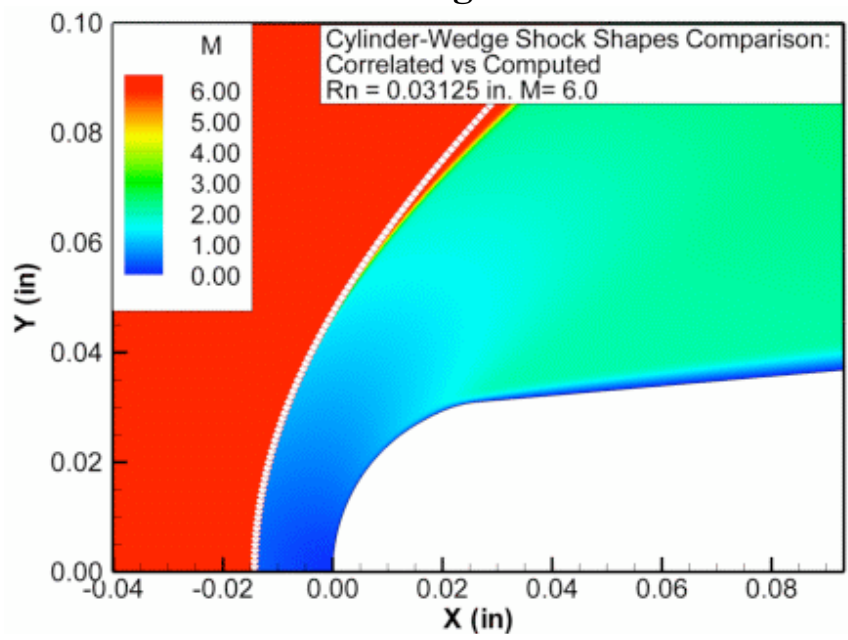

Figure 4. Comparison of computed and correlated ${ }^{21}$ bow shock shape for sphere-cone and cylinder-wedge. White line represents the correlated bow shock shape. 


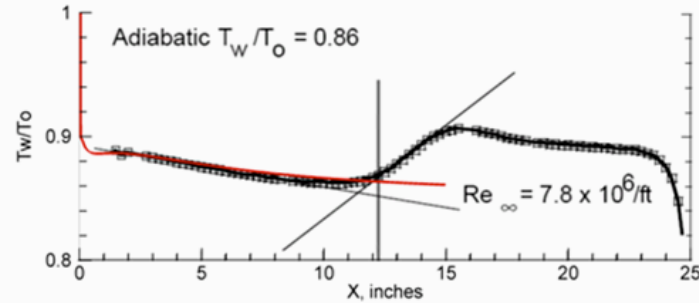

a) $\mathrm{Rn}=0.0625$ in

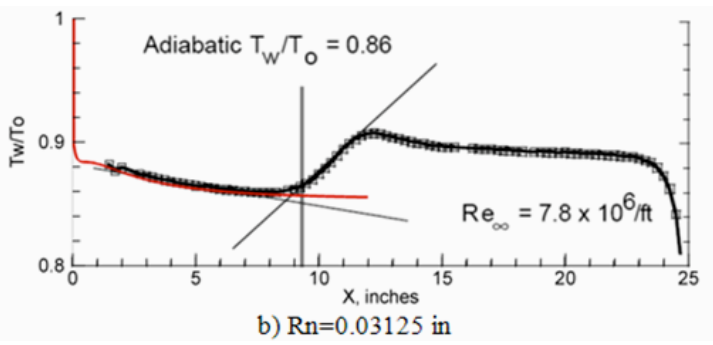

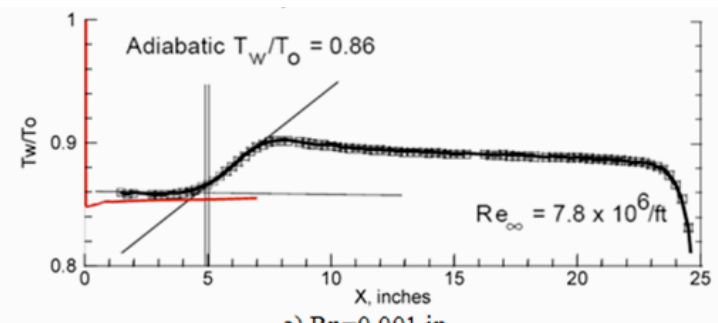

c) $\mathrm{Rn}=0.001$ in

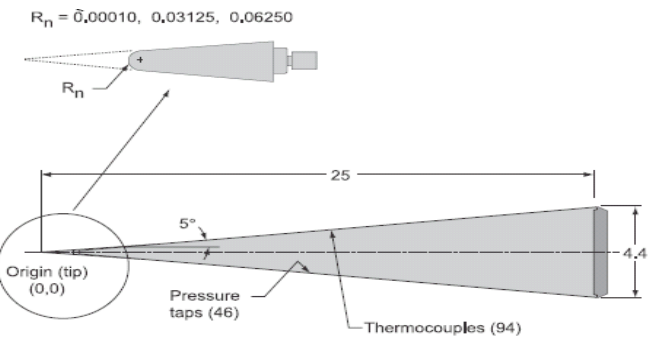

(d)

Figure 5. Comparison of computed wall to total temperature ratio with conventional wind tunnel data ${ }^{15}$ for different nose radii of 5 degree straight cone. (a) $R n=0.0625$-in, (b) $R n=0.03125$-in, (c) $R n=0.001$-in (d) Schematic diagram of the experimental mode ${ }^{15} \cdot M_{\infty}=6.0, \operatorname{Re}=7.8 \times 10^{6} / f t, T_{0}=475^{\circ} \mathrm{F}, P_{0}=475 \mathrm{psi}$

\section{Cone}
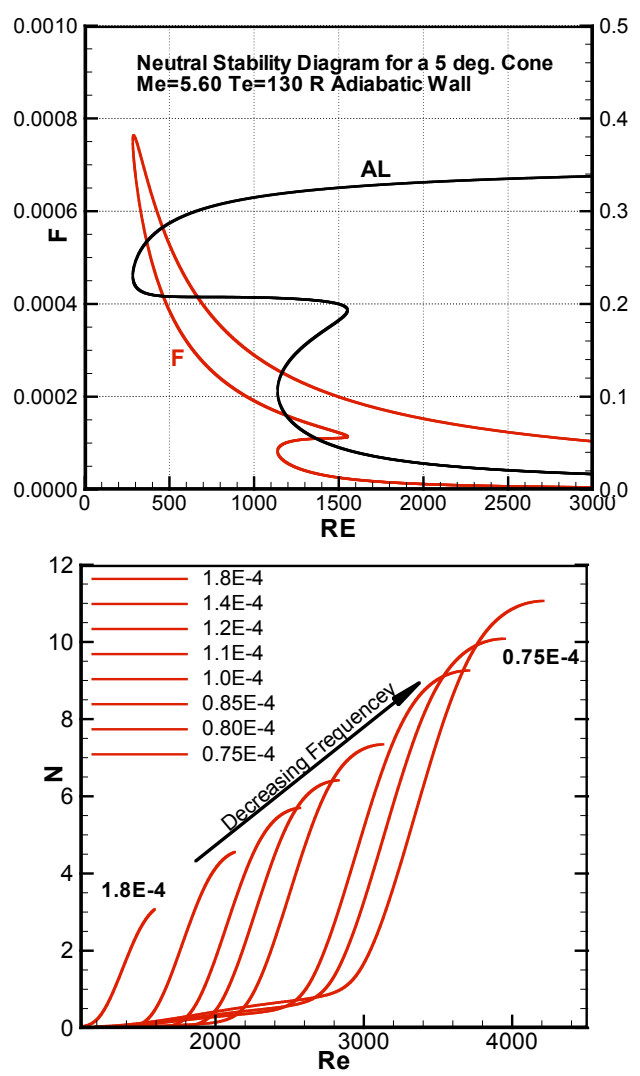

(a)

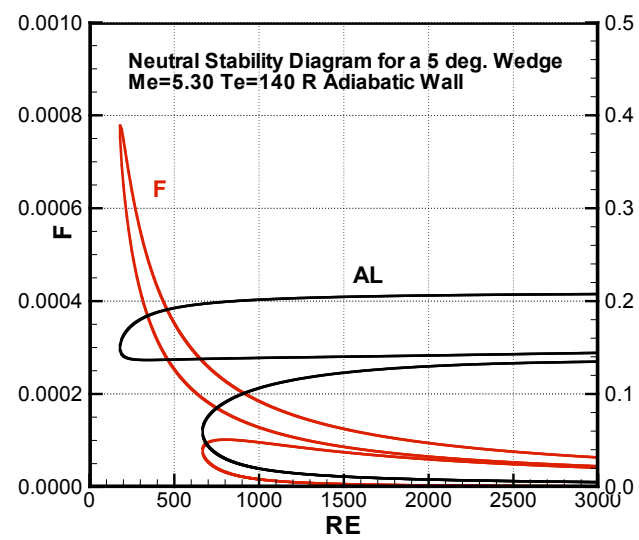

(c)

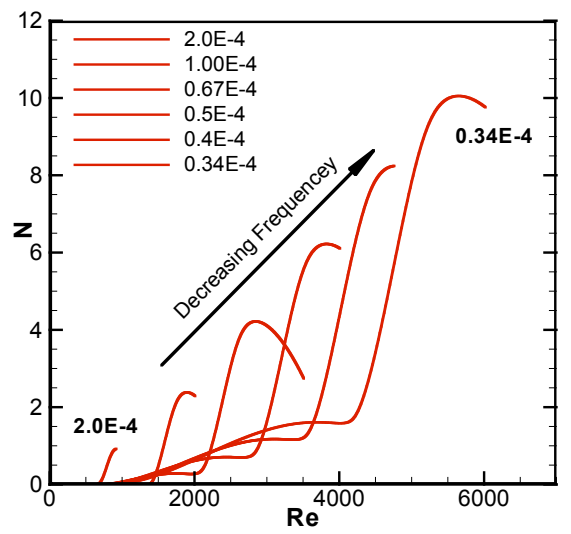

(b)

Figure 6. Neutral stability diagram and variation of wave number with Reynolds number. (a) Cone, (b) Wedge (c) N-Factor curves for increasing frequency for cone and (d) for wedge. 


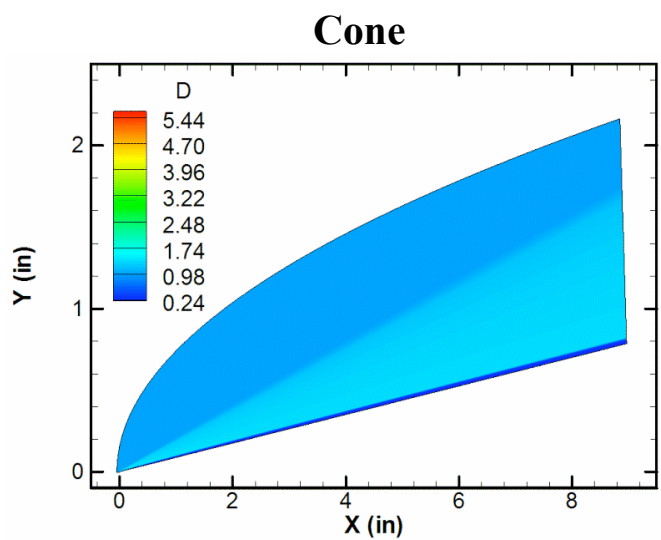

(a)
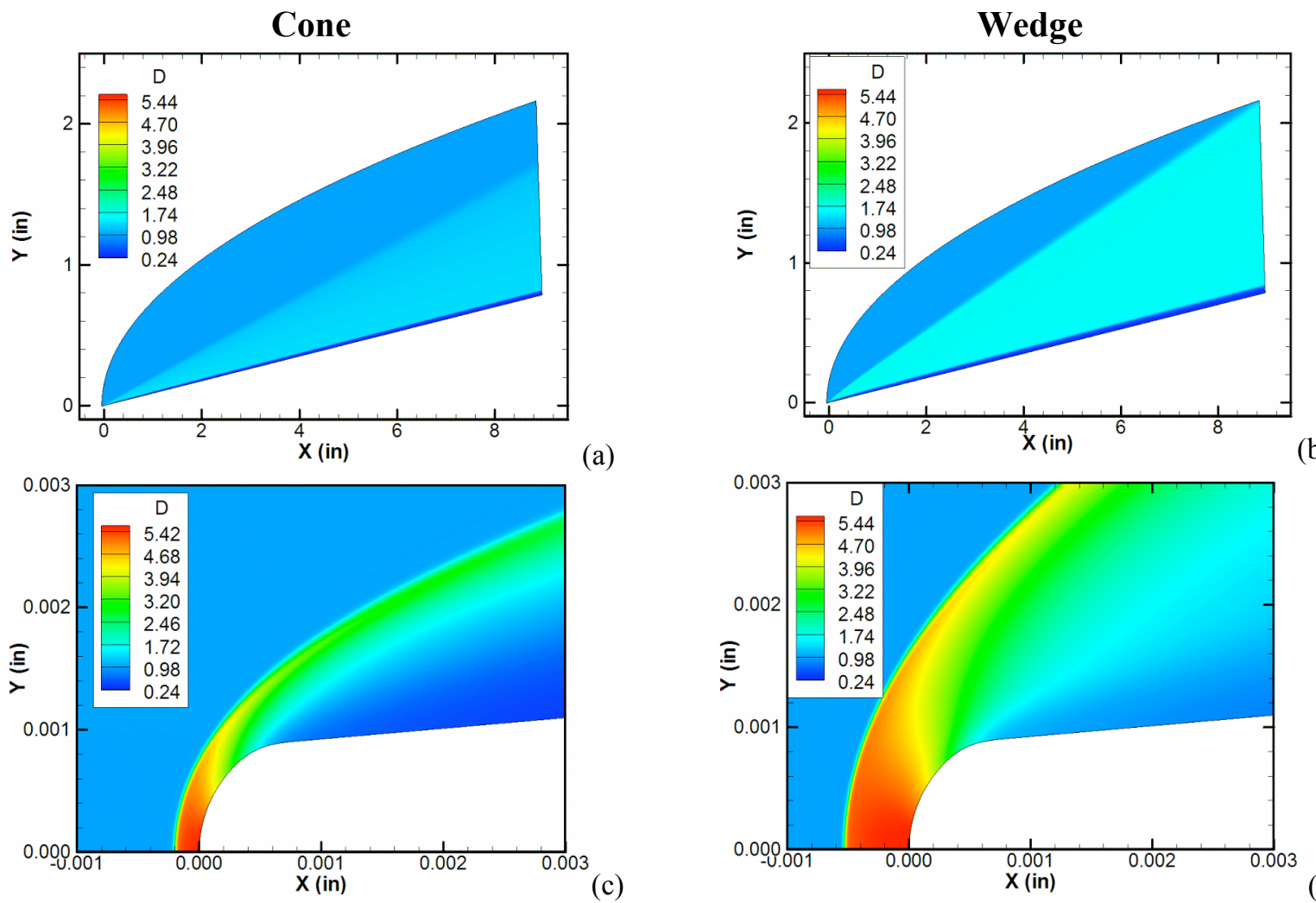

(c)
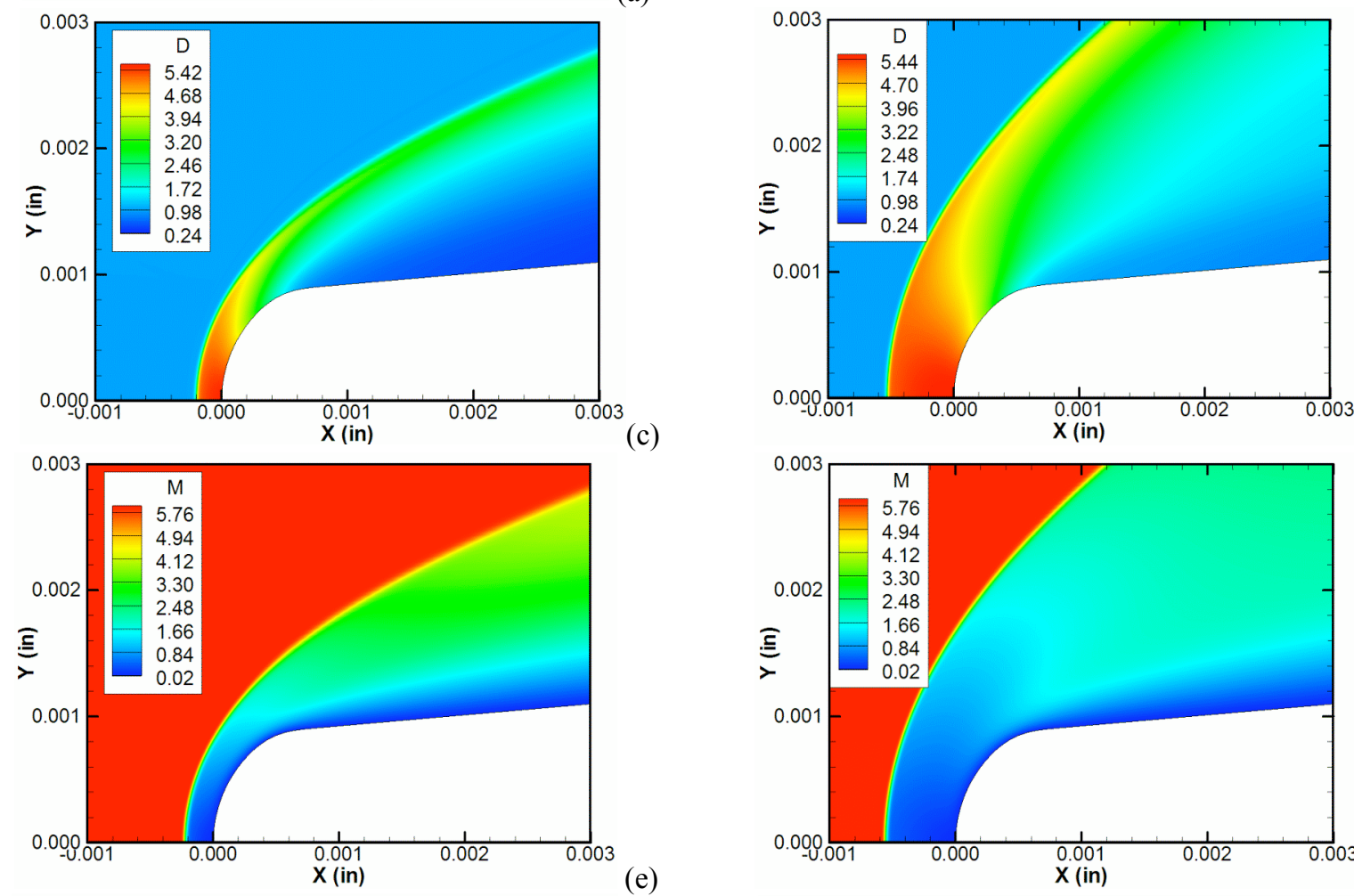

(e)
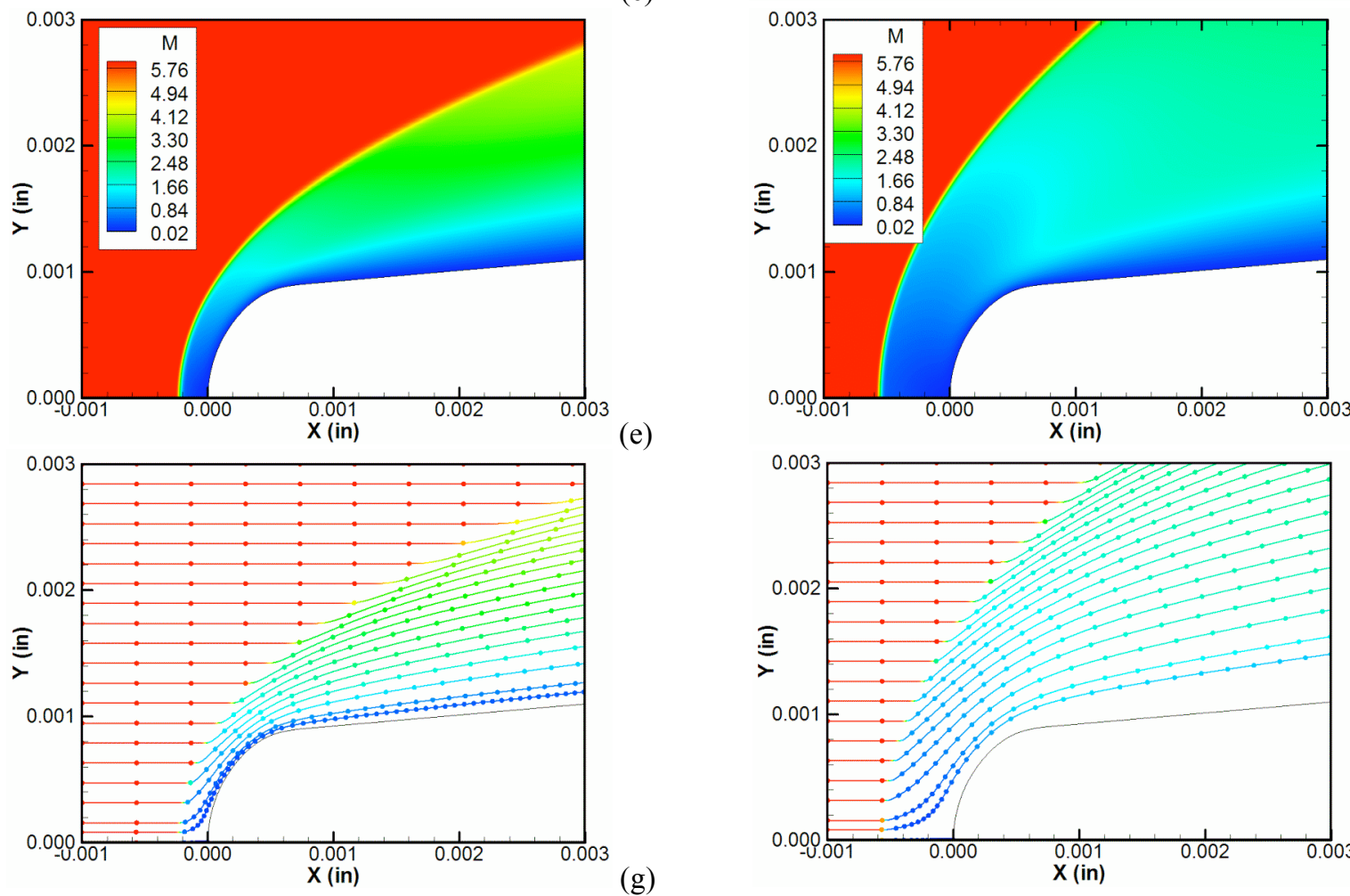

(g)

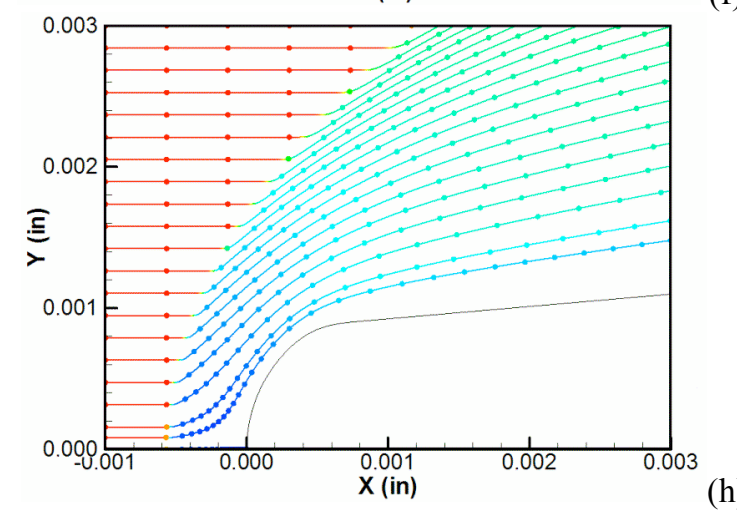

Figure 7. Comparison of mean flows of cone and wedge for Mach 6.0. Cone results are on the left and wedge results are on the right. (a), (b) Density contours in whole domain (c), (d) density contours, (e), (f) Mach contours, (g), (h) streamlines patterns near leading edge. 
Cone
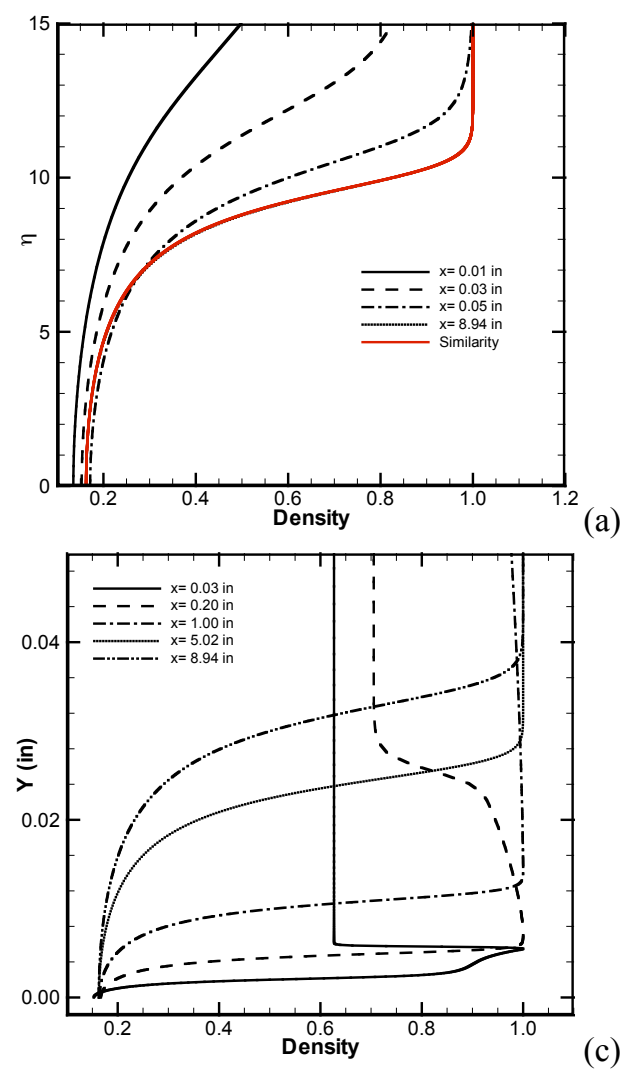

Wedge
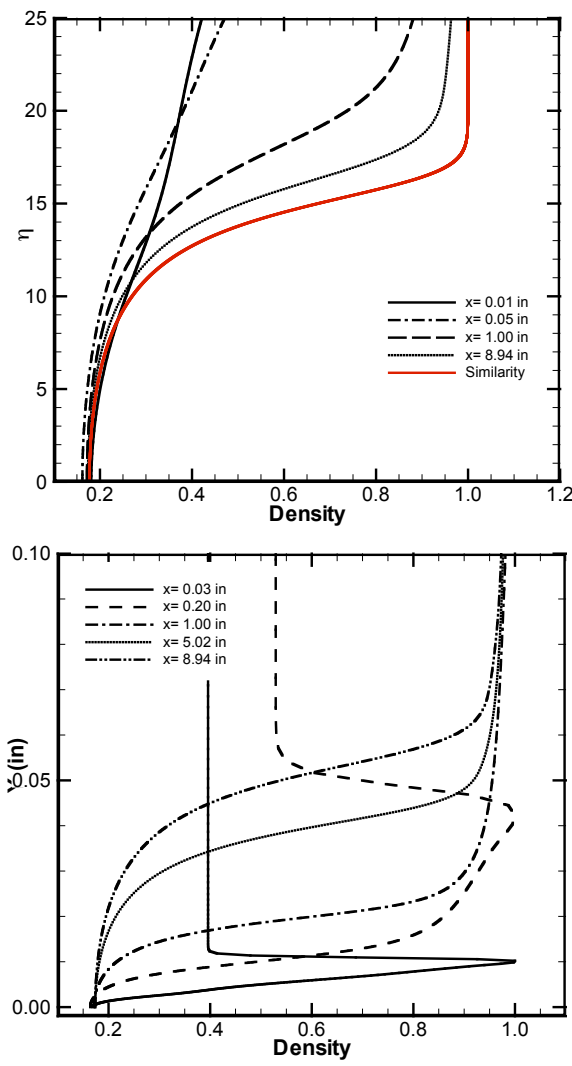

(b)

(d)

Figure 8. (a), (b) Mean density profiles at different $X$ locations using similarity coordinates for cone and wedge respectively. (c), (d) density profiles versus $Y$ coordinate for cone and wedge.
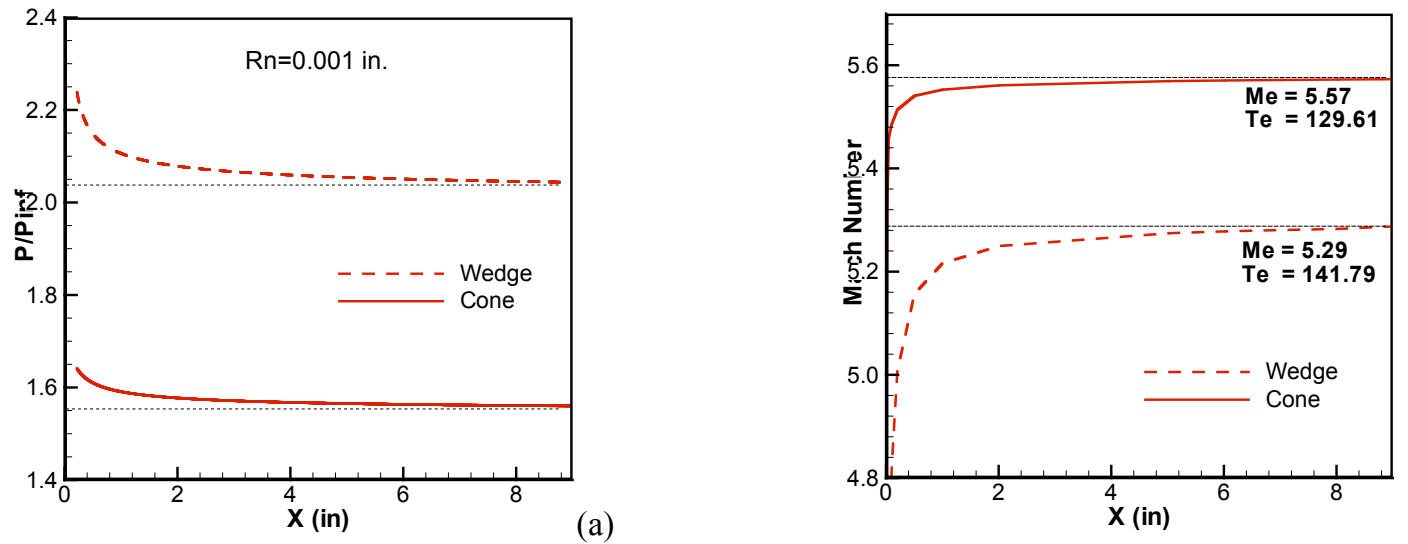

(a)

Figure 9. (a) Comparison of mean flow pressure distribution along the surface for wedge and cone. (b) Comparison of Mach number distribution at the edge of the boundary layer for wedge and cone. 

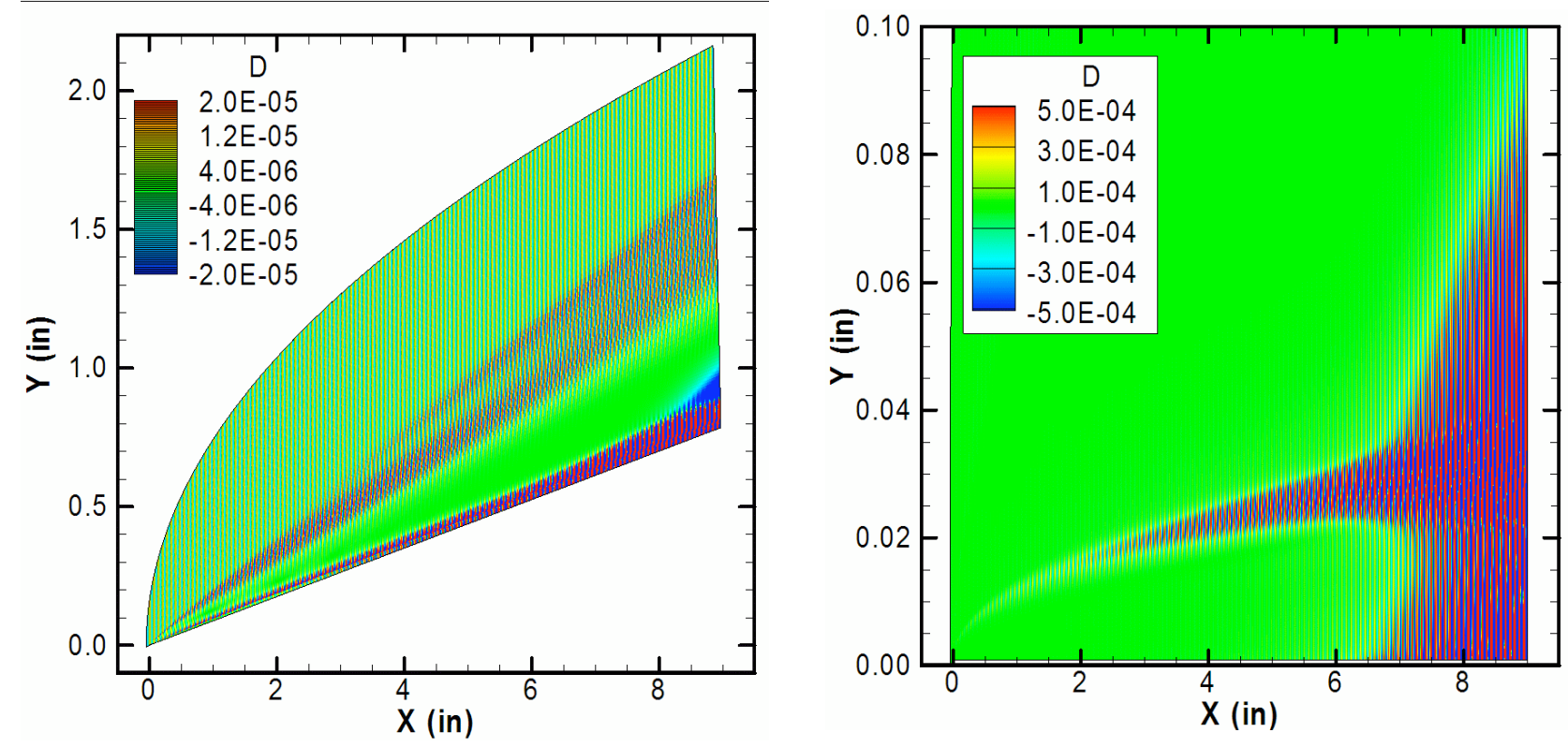

Figure 10. Contours of the unsteady density fluctuations due to the interaction of slow acoustic wave with a blunt cone: $F=1.2 \times 10^{-4}$. (a) Whole domain. (b) Fluctuations in the boundary layer. Computational domain rotated 5 degrees clockwise to show density fluctuations clearly.
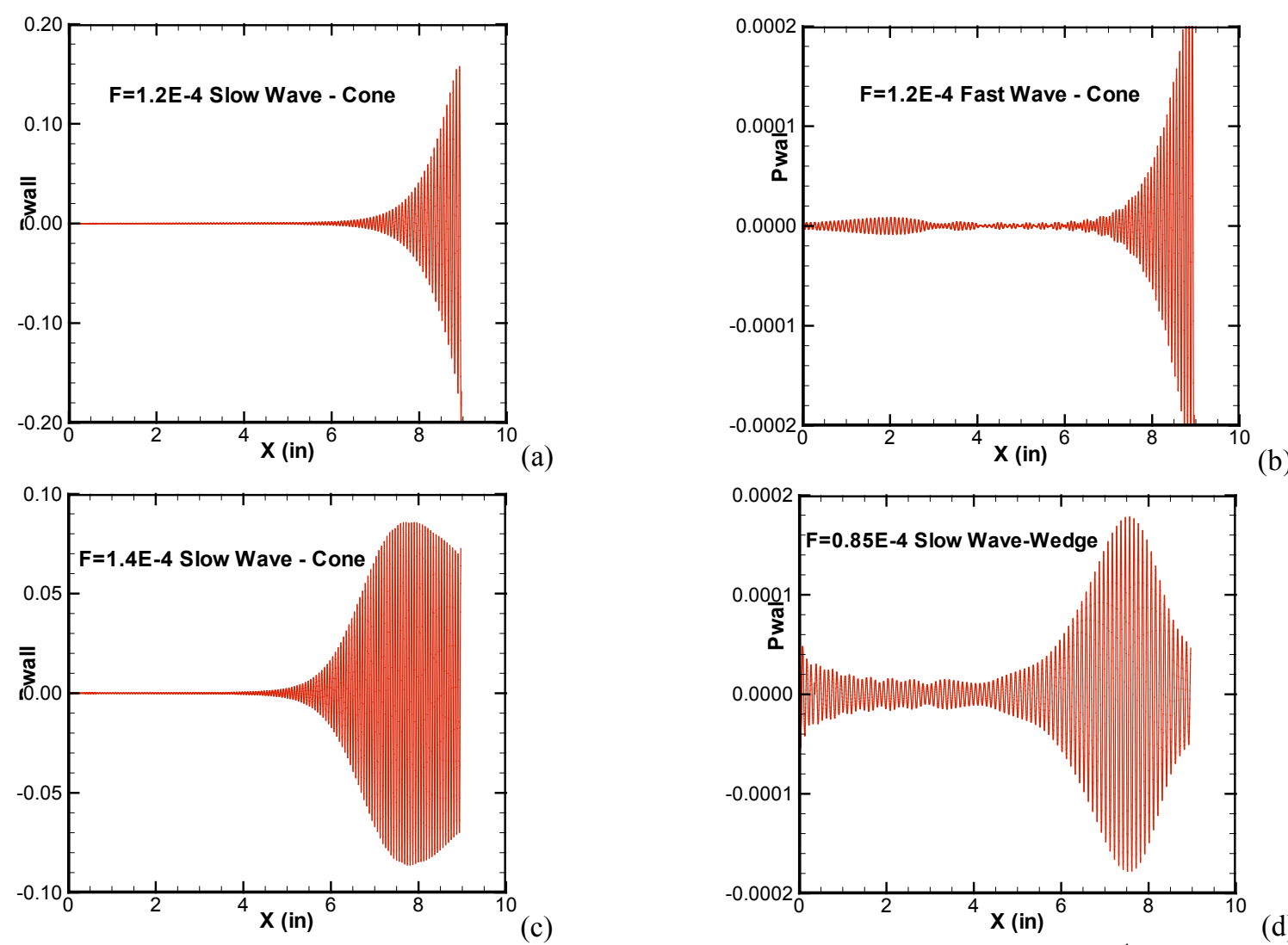

Figure 11. Wall pressure fluctuations generated by slow and fast acoustic modes. (a) $F=1.2 \times 10^{-4}$ Slow Mode-

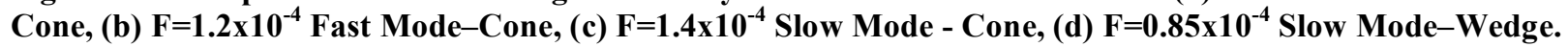



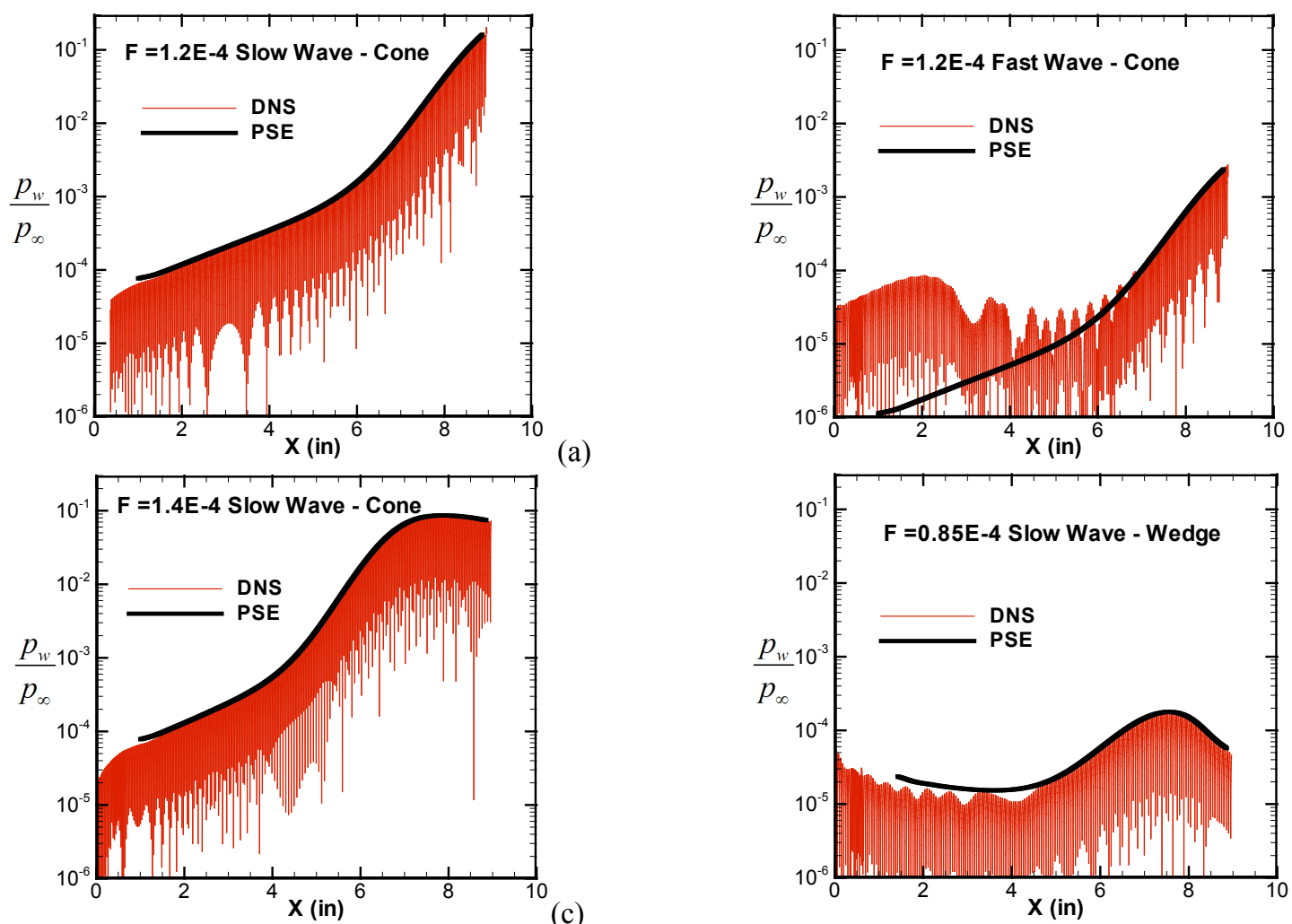

(b)

(c)

Figure 12. Amplitude of the pressure fluctuations at the wall and comparison with the PSE. (a) $F=1.2 \times 10^{-4}$ Slow Mode, (b) $F=1.2 \times 10^{-4}$ Fast Mode, (c) $F=1.4 \times 10^{-4}$ Slow Mode, (d) F=0.85x10 ${ }^{-4}$ Slow Mode.

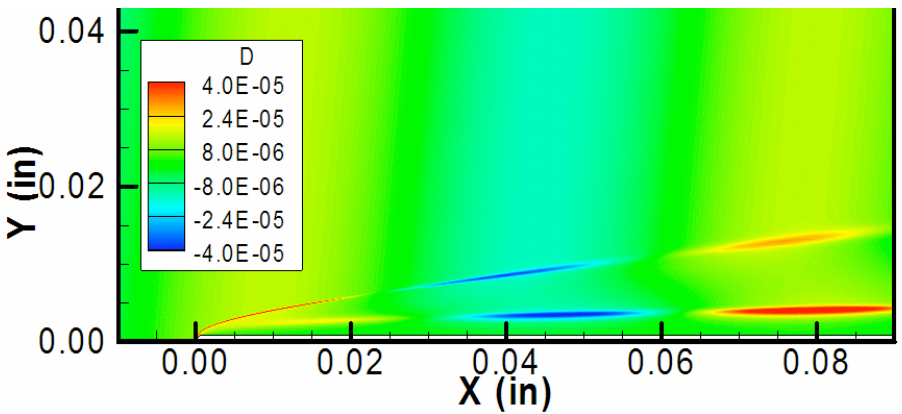

(a)

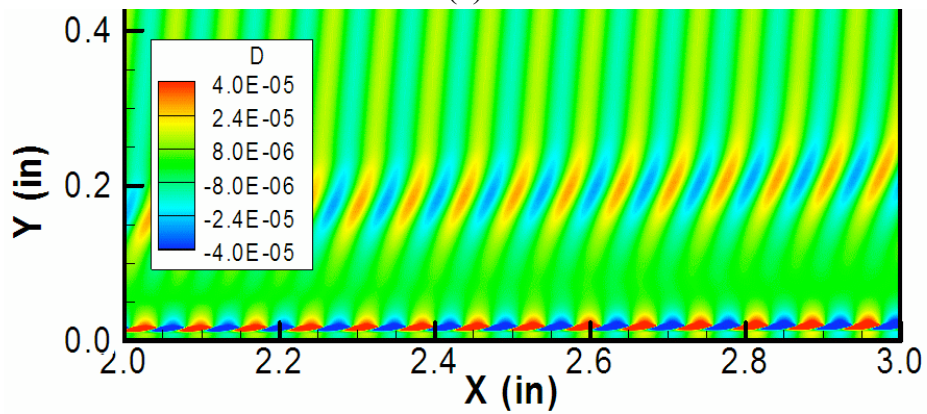

(c)

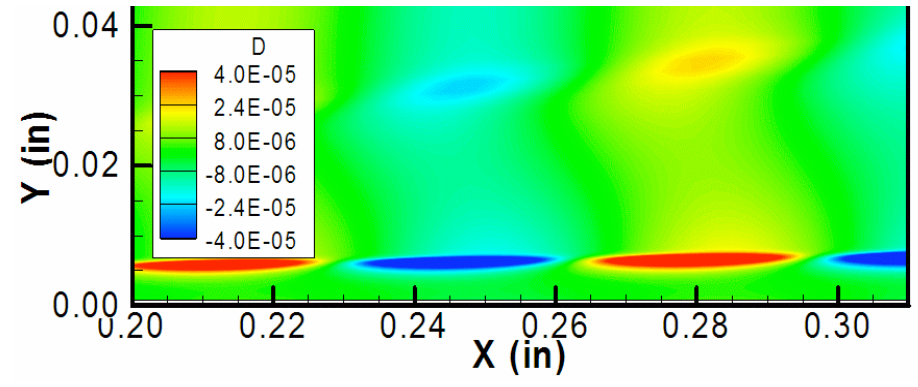

(b)

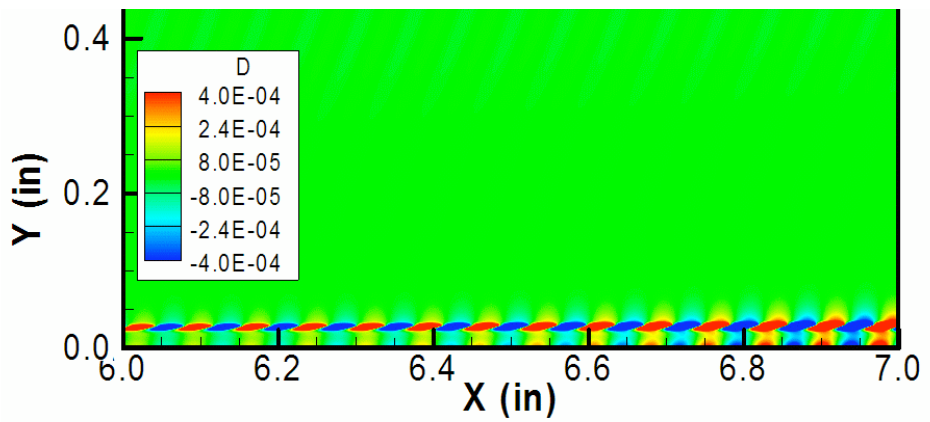

(d)

Figure 13. Contours of unsteady density fluctuations inside the boundary layer. $F=1.2 \times 10^{-4}$ Slow $\mathrm{Mode}^{-C o n e}$. $\mathrm{R}_{\mathrm{n}}=\mathbf{0 . 0 0 1}$ in. Computational domain rotated 5 degrees clockwise to show fluctuations in a better way. 